\title{
Uso e interés por las redes sociales entre las personas mayores desde una perspectiva de género. Un estudio en las universidades de mayores andaluzas
}

\section{Extracto:}

\section{Sumario}

1. Introducción

2. Método

3. Resultados

4. Conclusiones

5. Bibliografía

Fecha de entrada: 28-09-2016

Fecha de aceptación: 14-02-2017

Los datos ofrecidos en el presente artículo surgen de una investigación realizada a nivel autonómico en las diferentes universidades de mayores andaluzas. Uno de los propósitos de este estudio es conocer la situación de las personas mayores en las redes sociales en función del género. Participaron un total de 294 alumnos pertenecientes a los programas provinciales de las universidades de mayores. Para ello, se confeccionó un cuestionario tras una revisión de literatura y tomando como referente investigaciones realizadas con la temática de estudio. Los resultados indican que la brecha de género entre el colectivo prácticamente ha desaparecido al igual que en la generación más joven. Ambos géneros exponen las mismas preocupaciones al usar las redes sociales y coinciden también en los beneficios que les proporciona su uso, en las herramientas utilizadas o en los motivos que les llevaron a formar parte de las mismas. Sí se encuentran diferencias significativas entre hombres y mujeres con respecto al interés, el fin con el que se utilizan, la frecuencia de uso y los dispositivos de conexión utilizados.

Palabras clave: redes sociales, personas mayores, género, Web 2.0.

\footnotetext{
1 J. Barroso Osuna, profesor titular del Departamento de Didáctica y Organización Educativa de la Facultad de Ciencias de la Educación de la Universidad de Sevilla.

2 S. Aguilar Gavira, profesora sustituta interina del Departamento de Didáctica de la Facultad de Ciencias de la Educación de la Universidad de Cádiz.
} 


\section{Use and interest}

\section{in Social \\ networks}

\section{among older people \\ from a gender perspective.}

\section{A study in the andalusian senior people universities}

\section{Abstract:}

The data provided in this article arise from an investigation carried out as a regional level in the different andalusian senior people universities. One of the purposes of this study is to know the situation of senior people in social networks according to gender. A total of 294 students belonging from provincial programs of the different andalusian senior people universities participated. A questionnaire was elaborated, after a review of the literature and taking as a reference researches carried out about the subject of the study. The results indicate that the gender gap between the collective has practically disappeared as in the younger generation. Likewise, both genders express the same concerns as being part of social networks, benefits of use, reasons why they decided to be part of them or tools used to join. There are significant differences about interest, purpose, frequency of use and connection devices used between men and women.

Keywords: social networks, older people, gender, Web 2.0.

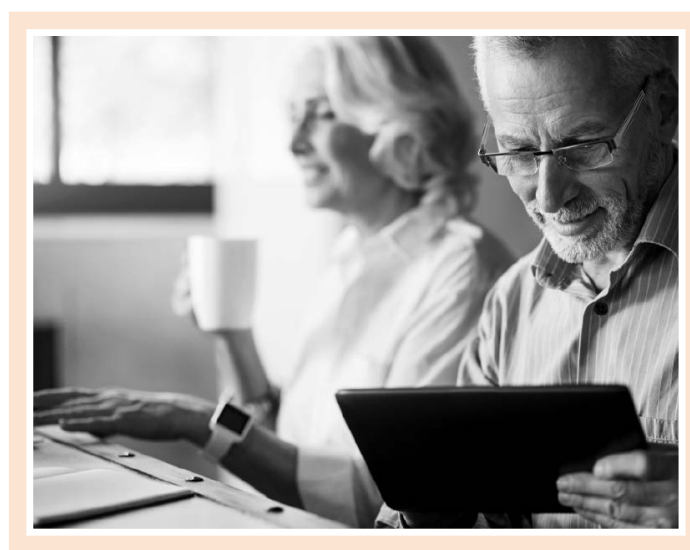

\section{INTRODUCCIÓN}

La eminente evolución tecnológica nos lleva a la denominada «Web 2.0», o «software social», caracterizada por la aparición de numerosas herramientas que han supuesto nuevas posibilidades de apertura a la información, a la formación, y que han ampliado las posibilidades de comunicación y de conexión entre las personas, facilitando así la colaboración, la interacción y el intercambio social orientados a aprender y a alcanzar un objetivo común (Solano y Bernal, 2011). Una cultura de la participación, surgida con la Web 2.0, que paraAparici y Osuna (2013) implica una serie de cambios, como son menores barreras para la expresión ciudadana, un soporte a la creatividad, mayores posibilidades de compartir el conocimiento, mayor valor de las experiencias y mayor valoración de las opiniones de los iguales, favoreciendo la construcción de una sociedad más justa y democrática. En el mundo de las redes sociales, «el nivel de conocimiento entre usuarios puede ser disímil, pero todos disponen de la misma posición u oportunidad para ofrecerlo» (Barroso y Aguilar, 2015, pág. 18).

Esta Web 2.0 supone un cambio social que De Clercq (2009) sintetiza en varios puntos:

- El bajo coste de la infraestructura para producir y publicar contenidos.

- La democratización de la opinión pública, siendo los propios usuarios los que toman la decisión al compartir, difundir, votar y comentar con sus mensajes y enlaces.

- El consumo de la información, que es filtrada por el propio usuario y se canaliza en distintas fuentes. 
Los cambios experimentados con la Web 2.0, donde los datos generados y su transmisión son mucho mayores, también nos conduce a un novedoso perfil de usuarios que presentan nuevas actitudes y que usan las herramientas de manera diferente a como se venía haciendo, determinando con ello la propia evolución social y tecnológica. Entre las características más relevantes de estos nuevos usuarios 2.0 podemos destacar las siguientes (Edukanda, 2016):

- Mayor participación social. Son usuarios que aportan, difunden, comparten y colaboran valiéndose de las herramientas que la Web 2.0 les proporciona, como wikis, redes sociales, blogs, sindicación de contenidos, marcadores sociales, etc. Entre sus objetivos destaca colaborar en la generación del conocimiento colectivo.

- Son usuarios 2.0, que conocen las utilidades que estas herramientas les pueden proporcionar, que poseen una cultura tecnológica elevada y que saben manejar la información y transformarla en conocimiento.

- Amplían sus relaciones personales, profesionales, etc., mediante herramientas que les permiten ampliar esta comunicación, conocer nuevos contactos e incluso modificar la forma de interactuar con el entorno.

- Es frecuente que dichos usuarios dominen términos tecnológicos, tecnicismos, etc., y que manejen el idioma inglés para poder utilizar aquellas herramientas, aplicaciones y servicios ofrecidos por internet y que no están traducidos al castellano (Ortega, 2007).

Según los datos presentados por Statista (2016), entre 2014 y 2018, el número de usuarios de las redes sociales españolas presentará un claro aumento, con 19 millones de usuarios previstos para 2018, frente a los 17 millones de usuarios actuales. Asimismo, este mismo portal de estadísticas recoge que las redes sociales preferidas por los usuarios son Facebook y WhatsApp, con una diferencia de alrededor de 30 puntos porcentuales respecto a YouTube, la tercera red social seleccionada por los ciudadanos.

La evolución tecnológica y el uso masivo de las redes sociales han venido acompañados de ciertas diferencias, convirtiéndose la desigualdad de género en una de las preocupaciones en la sociedad de la información y el conocimiento, y, por ende, se ha convertido en objeto de estudio de numerosos investigadores desde los inicios. Según el Instituto de la Mujer y para la Igualdad de Oportunidades (2015), analizar la situación de las re- des sociales desde una perspectiva de género conlleva la necesidad de diferenciar la existencia de tres brechas digitales, coexistentes, aunque de carácter y alcance diferente:

- Brecha de acceso a la tecnología, determinando la incorporación y la participación o no de las mujeres en la sociedad de la información en igualdad de oportunidades.

- Brecha de uso, relacionada con la utilización que se hace de las tecnologías de la información y la comunicación (TIC), marcando el grado de incorporación efectiva y las diferencias de intensidad, frecuencia, usos y habilidades.

- Uso y aprovechamiento de las aplicaciones más innovadoras de internet.

Si en un primer momento el acceso a las TIC se había caracterizado por alcanzar una mayor tasa de adopción masculina, con una escasa presencia por parte de las mujeres, con el paso de los años esa diferencia se ha ido mitigando entre los más jóvenes, alcanzando un acceso similar entre ambos géneros e incluso, en algunas redes sociales, superior por parte de las mujeres (Barragán y Ruiz, 2013; Gil, Vitores, Feliu y Vall, 2011). Sin embargo, aunque se haya logrado una igualdad en cuanto al acceso, según investigaciones realizadas, se siguen detectando en el tiempo importantes diferencias en relación con la frecuencia de uso, el aprovechamiento, las habilidades digitales, etc. A medida que se requieren mayores destrezas técnicas, disminuye el uso por parte de las mujeres. Esto quizás se deba a una visión preconcebida sobre la tecnología entre hombres y mujeres, según la cual los primeros ven la tecnología como algo positivo, mientras que las segundas lo hacen de forma más negativa (Jackson, Ervin, Gardner y Schmitt, 2001; Romero, 2011).

La evolución tecnológica y el uso
masivo de las redes sociales han
venido acompañados de ciertas
diferencias, convirtiéndose la
desigualdad de género en una de las
preocupaciones en la sociedad de la
información y el conocimiento
masivo de las redes sociales han venido acompañados de ciertas diferencias, convirtiéndose la desigualdad de género en una de las información y el conocimiento 
Estudios realizados en 2013 nos indican un predominio de las mujeres en el uso de las redes sociales, principalmente de Facebook, donde participan el $69,5 \%$ de las internautas (11 puntos porcentuales más que los hombres). Una situación que difiere respecto a Tuenti y Twitter, en las que hay una mayor influencia del género masculino (Fundación Telefónica, 2013). Una investigación llevada a cabo en Andalucía por Colás, González y De Pablos (2013) sobre las motivaciones de los jóvenes a la hora de utilizar las redes sociales y la frecuencia de uso de las mismas constata que no existen diferencias significativas entre géneros respecto a la frecuencia de uso, aunque sí se perciben diferencias entre ellos con relación a las motivaciones: en las chicas, las motivaciones son más de tipo relacional/social, mientras que en los chicos dichas motivaciones son más de reconocimiento personal (las chicas usan más las redes sociales para hacer amigos y los chicos las utilizan porque les gusta saber lo que dicen sus amigos y ver las fotos que estos suben, y porque entrar en las redes sociales les hace sentir bien cuando están tristes).

Si investigamos la situación de las personas mayores con respecto a estos nuevos medios y servicios tecnológicos, son varios los estudios que han verificado las ventajas que supone su uso para este colectivo, entre ellos, el realizado en 2009 en la Universidad de California por el profesor Gary Small en el que participaron 24 personas entre 55 a 78 años. Según los resultados de dicho estudio, navegar por internet para buscar información de interés o simplemente para comunicarnos con otras personas es una actividad que nos obliga a activar centros clave del cerebro humano, mejorando la memoria, por lo que se constata que el cerebro no pierde la capacidad de aprender por haber envejecido.

Autores como Jiménez (2016) afirman que las nuevas tecnologías suponen una herramienta eficaz para el envejecimiento activo y, por ende, para la calidad de vida de los mayores, puesto que su uso hace más fácil y cómodas muchas de las tareas que realizamos en nuestra vida cotidiana. Según Barroso y Aguilar (2016, pág. 230), «su participación en ellas permite superar ciertas discapacidades asociadas con el envejecimiento, entre las que podemos encontrar el aislamiento, permitiéndoles, a su vez, permanecer en constante evolución con la sociedad, así como compartir intereses, preocupaciones o necesidades».
La presencia de las personas mayores en las redes sociales les aporta una mayor calidad de vida, como expone la Organización Mundial de la Salud (OMS) (2001), convirtiéndoles en ciudadanos activos y mejorando sus capacidades mentales, como la memoria.

Según Fernández y Fueyo (2014, pág. 161), la calidad de vida del individuo está influida «de modo complejo por la salud física del sujeto, su estado psicológico, su nivel de independencia, sus relaciones sociales, así como su relación con los elementos esenciales del entorno, entre los que se pueden contar la comunicación en red con sus iguales y familiares, con el movimiento organizado de mujeres o con la sociedad civil en general, así como el acceso a la información y a la participación sociopolítica a través de las redes sociales». Entre los estudios existentes relativos a las personas mayores y a las redes sociales como Facebook, podemos mencionar los realizados por Bell et al. (2013). Tras los resultados obtenidos en su investigación, los autores inciden en que las personas mayores de 60 años que son usuarios de una red social tienen valores más altos en cuanto a satisfacción social y confianza en la tecnología que aquellos otros mayores que afirman no utilizarla.

Las redes sociales brindan un amplio abanico de posibilidades a los intereses y a las necesidades manifestadas por los propios mayores, como son «la comunicación, ya que les relaciona con el entorno, y también la información, porque les mantiene al día en su curiosidad de saber y conocer» (Sebastián y Martínez, 2013, pág. 3). Sin embargo, según los resultados ofrecidos por Abellán y Pujol (2015), en España, uno de los países más longevos de Europa, a pesar de que el colectivo ha aumentado su uso con respecto a años anteriores, sobre todo entre las mujeres, aún sigue existiendo una brecha digital entre las personas mayores. Según estos autores, a partir de los 55 años, y a medida que aumenta la edad del usuario, se observa un importante descenso del uso de internet y del interés por las redes sociales con respecto a los más jóvenes de nuestra población, por lo que su penetración en esta sociedad tecnológica está siendo mucho más lenta. Facilitar el acceso, motivar y formar en el uso de las redes sociales a dicho grupo etario supone promover la inclusión social de nuestros mayores. Estudios como los de Braun (2013) y Dias (2012) señalan las principales motivaciones e intereses de las personas mayores en el uso de las tecnologías digitales, así 
como las posibles barreras con las que se encuentra dicho colectivo para formar parte de las mismas.

Asimismo, y en contraposición a los más jóvenes, en el caso de las personas mayores, la brecha de género sigue patente (Instituto Nacional de Estadística [INE], 2016a, 2016b; Abellán, Vilches y Pujol, 2014; Abellán y Pujol, 2015). Los prejuicios y mitos que se expanden tanto en el entorno familiar como social en relación con la complejidad tecnológica influyen negativamente en la motivación de las mujeres mayores con respecto a sus posibilidades para formar parte de la sociedad de la información y el conocimiento.

Según la opinión de un grupo de mujeres que participaron en un estudio sobre la brecha de género realizado por Fernández y Fueyo (2014), la causa principal de la misma se debe a la ausencia de corresponsabilidad en el hogar, donde las mujeres, al ocuparse de la familia y de las tareas domésticas, disponen de menos tiempo para participar en las redes sociales, seguido de otras variables, como la falta de formación e información, asociadas con la escasez de oportunidades que han tenido en el pasado para formarse o con que la mujer que está en casa no tiene las mismas posibilidades de acceso a un ordenador que el hombre, quien puede disponer de este y de acceso a internet en su lugar de trabajo.

Además, se han constatado diferencias significativas no solo en cuanto al acceso, sino también con respecto a las herramientas utilizadas entre hombres y mujeres mayores. Mientras que aplicaciones consideradas más fáciles o cotidianas, como el e-mail, son utilizadas igualmente por ambos géneros, en el caso de otras actividades, como la descarga de softwares, la banca electrónica o la lectura de noticias online, son usadas con mayor frecuencia por los hombres que tienen profesiones de mayor responsabilidad y formación (Peral, Arenas y Ramón, 2013). Todo ello nos hace ver que no se trata solo de poner en marcha políticas para facilitar el acceso a la tecnología de nuestros ciudadanos, sino de ir más allá, algo mucho más importante y que supone una mayor implicación, es decir, ofrecer los conocimientos y las habilidades necesarias para que dicho colectivo pueda, al igual que el resto de ciudadanos, comunicarse, opinar y construir conocimiento en la red.

La necesidad del contacto habitual con la familia y los amigos explica el interés y la motivación del uso de las redes sociales por parte de las personas mayores
(Fernández y Fueyo, 2014; Fundación Vodafone, 2012; Requena, Pastrana y Salto, 2012), seguido del acceso a la información, permanecer al corriente de las noticias y de la actualidad política y social. Estudios llevados a cabo exclusivamente entre las mujeres mayores, como los de Fernández y Fueyo (2014), así como investigaciones realizadas con hombres y mujeres, como la de Vilte, Saldaño, Martín y Gaetán (2013), han evidenciado que la familia (los hijos) suele ser el primer nexo de unión entre los mayores y la tecnología, bien de forma directa, ayudándoles ante sus dificultades 0 temores, o bien de forma indirecta, despertando su interés al introducir el uso de las tecnologías en casa. Igualmente, las personas mayores que forman parte de redes sociales son asiduos a las mismas, accediendo en su mayoría todos los días (Fernández y Fueyo, 2014; Vilte, Saldaño, Martín y Gaetán, 2013).

Sí se encuentran diferencias significativas dentro del colectivo al utilizar las redes para relacionarse con personas de su misma profesión, siendo estas usadas con dicho fin por las personas mayores que poseen un mejor nivel formativo. Por otro lado, el colectivo no demanda redes sociales adaptadas a las necesidades, edad e intereses particulares, sino que se señala el uso de herramientas generalistas, donde su «mundo» vaya más allá de aquellos con los que tienen aparentemente más identidad (Fundación Vodafone, 2012).

Aunque el teléfono móvil es una de las tecnologías de las que actualmente dispone prácticamente la totalidad de la población y que es considerada como fundamental, el uso de internet a través de él alcanza resultados muy diferentes. Atendiendo al perfil demográfico, podemos decir que el mayor grado de penetración a internet a través del teléfono móvil se da en edades comprendidas entre los 16-24 años, con un $66,7 \%$; en personas mayores, con edades comprendidas entre los 55-64 años, solo alcanza un valor del 20,4\%; y entre los 65-74 años, solo tiene una penetración del $12 \%$; porcentaje este último que coincide, según investigaciones realizadas, con aquellos que son usuarios habituales de internet (Fundación Telefónica, 2013). Otros estudios ratifican dichos datos, puesto que, en el caso de la población mayor, el ordenador es el medio más utilizado y casi el único por ahora para conectarse a internet, quedando muy lejos actualmente el uso de otro tipo de dispositivos de conexión, como el teléfono móvil o la PDA, dada la escasa frecuencia alcanzada (Martínez, Delerue y Silva, 2013). 


\section{MÉTODO}

El presente estudio se ha abordado desde una metodología de investigación cuantitativa, de tipo descriptiva, a través de la cual se «recolectan datos sobre diversos conceptos (variables), aspectos, dimensiones o componentes del fenómeno que hay que investigar» (Hernández, Fernández y Baptista, 2006, pág. 81), para así, posteriormente, detallar o describir la situación en la que se encuentra el objeto de análisis. La técnica utilizada para la recogida de información sobre las variables que hay que medir ha sido un cuestionario.

Son cuatro las dimensiones que han constituido nuestro cuestionario y que ofreceremos a lo largo de este artículo. En la primera dimensión, se pretendía recoger información de las personas encuestadas relativa al género, la edad, el estado civil o el nivel de estudios. Asimismo, nuestra segunda dimensión tenía como objetivo conocer la visión de este colectivo sobre las redes sociales en función del género y estaba constituida por una serie de ítems, como interés por conocerlas y aspectos en los que ellos consideraban que su uso les podría beneficiar o perjudicar. Las dos últimas dimensiones, relacionadas con el conocimiento y el uso de las herramientas, pretendían conocer el número de sujetos que pertenecían a alguna red social en función del género, motivo de su uso, redes sociales utilizadas y fin de su uso, tiempo de uso que hacian de las mismas y dispositivos de conexión empleados.

\subsection{Objetivos del estudio}

Entre los objetivos planteados en nuestra investigación, y que presentamos en este artículo, está conocer si existen diferencias o no entre nuestros mayores con respecto a las redes sociales en función del género. Para ello, se analizó la variable "Género» con otras variables del estudio, como:

- Presencia en las redes sociales.

- Nivel de interés.

- Riesgos y beneficios que supone el uso de las redes sociales para las personas mayores.

- Frecuencia de acceso.

- Motivo por el que utilizan las redes sociales.

- Uso que se hace de las redes sociales.

- Redes sociales más utilizadas.

- Dispositivo de conexión.

\section{Entre los objetivos planteados en nuestra investigación [...] está conocer si existen diferencias 0 no entre nuestros mayores con respecto a las redes sociales en función del género}

\subsection{Participantes}

La muestra seleccionada quedó configurada por un total de 294 alumnos, de los cuales el $63,1 \%$ (188) eran mujeres y el $36,1 \%$ (106) hombres. Para su selección se empleó un muestreo no probabilístico intencional, es decir, aquel en el cual el investigador selecciona directa e intencionalmente la muestra debido a que tiene fácil acceso a la misma y es representativa de la población (Albert, 2006; Sabariego, 2004). El alumnado participante se encontraba matriculado en el tercer curso de los programas provinciales de las universidades de mayores de la Comunidad Autónoma de Andalucía (en concreto, en Sevilla, Cádiz, Málaga, Granada, Córdoba, Jaén y Almería).

El mayor número de alumnos de la muestra pertenecía a la Universidad de Sevilla ( $f=99 ; 33,7 \%$ ), seguido de Málaga ( $f=60 ; 20,4 \%$ ), Cádiz ( $f=36 ; 12,2 \%$ ), Almería y Jaén ( $f=29 ; 9,9 \%$ ), Granada ( $f=22 ; 7,5 \%$ ) $y$, por último, con el menor porcentaje, Córdoba $(f=$ $=19 ; 6,5 \%)$.

Respecto a su edad, el $52 \%$ de la muestra se encontraba en un intervalo de edad entre los 55-65 años, y el $45 \%$, entre los $65-75$ años, siendo muy reducido $(3 \%)$ el número de participantes que se situaba en un intervalo entre los 75-85 años. Además, la mayoría de ellos estaban casados. Si cruzamos el estado civil y el género, encontramos diferencias significativas. En el caso de los hombres, la opción «Divorciado» fue la segunda opción con mayor porcentaje alcanzado, mientras que, en el caso de las mujeres, esta fue la que menor porcentaje logró, siendo para ellas la opción «Viuda» la segunda opción con mayores porcentajes obtenidos. 
Nos encontramos ante una muestra con un nivel de formación bastante elevado, donde tan solo un $0,7 \%$ no tenía estudios y un $9,2 \%$ había cursado únicamente estudios primarios. En el resto de la muestra, el $22,1 \%$ eran diplomados, seguido por un $19,4 \%$ con título de bachillerato, un $17,3 \%$ con título profesional o título de técnico superior, un $14,3 \%$ con título de licenciado, un $13,9 \%$ con título de secundaria y un $3,1 \%$ con título de doctor. En el caso concreto del género masculino, sus mayores porcentajes se encontraban bajo la opción de licenciados, uno de los niveles de estudios con menor porcentaje alcanzado entre el género femenino. En el caso de las mujeres, la opción de diplomadas era la que alcanzaba los mayores porcentajes, siendo esta opción menor en los hombres. Las familias profesionales que aparecieron con mayor frecuencia entre nuestra muestra fueron educación ( $f=51 ; 17,3 \%)$, administración y gestión ( $f=47 ; 16 \%)$, ama de casa ( $f=39$; $13,3 \%)$, sanidad ( $f=35 ; 16 \%$ ) y comercio y marketing $(f=27 ; 19,2 \%)$. El resto de profesionales alcanzaron frecuencias y porcentajes muy reducidos, e, incluso, no obtuvieron ningún valor $(f=0 ; 0 \%)$ profesiones como energía y aguas y actividades fisicodeportivas.

\subsection{Instrumento}

El instrumento utilizado para analizar a las personas mayores con respecto a su papel en las redes sociales desde una perspectiva de género fue un cuestionario. Las fases que se llevaron a cabo para la construcción del instrumento fueron:

- Revisión de literatura y de cuestionarios que habían sido elaborados en anteriores investigaciones relacionadas con la temática.

- Elaboración de la primera versión del cuestionario.

- Validez del instrumento.

- Elaboración de la segunda versión del instrumento tras las apreciaciones obtenidas.

- Obtención del índice de fiabilidad.

- Aplicación del instrumento a la muestra seleccionada.

Para garantizar la validez de los contenidos y la aplicabilidad del instrumento en relación con la información que deseábamos obtener, este fue sometido a juicio de expertos, concretamente mediante el método de agregados individuales. Aunque se considere como una limitación el hecho de que los expertos no

\author{
Nos encontramos ante una \\ muestra con un nivel de \\ formación bastante elevado, \\ donde tan solo un $\mathbf{0 , 7} \%$ no tenía \\ estudios y un $9,2 \%$ había cursado \\ únicamente estudios primarios. \\ En el resto de la muestra, el \\ $\mathbf{2 2 , 1 \%}$ eran diplomados, seguido \\ por un $19,4 \%$ con título de \\ bachillerato, un $17,3 \%$ con título \\ profesional o título de técnico \\ superior, un $14,3 \%$ con título de \\ licenciado, un $13,9 \%$ con título \\ de secundaria y un $3,1 \%$ con \\ título de doctor
}

pueden intercambiar sus opiniones, puntos de vista y experiencia, sin embargo, esta limitación puede ser precisamente su ventaja, evitando los sesgos de los datos ocasionados por conflictos interpersonales, presiones entre los expertos, etc. (Corral, 2009). Se seleccionó a un grupo de expertos compuesto por profesores de Tecnología Educativa y Nuevas Tecnologías de la Universidad de Sevilla y por miembros del Panel 
Internacional de Investigación en Tecnología Educativa (PI2TE) de la asociación EDUTEC. Para su selección utilizamos el coeficiente de competencia experta o coeficiente $\mathrm{K}$, obtenido tras la aplicación de la fórmula $\mathrm{K}=1 / 2(\mathrm{Kc}+\mathrm{Ka})$, donde $\mathrm{Kc}$ es el «coeficiente de conocimiento» o información que tiene el experto acerca del tema y Ka es el denominado «coeficiente de argumentación» o fundamentación de los criterios de los expertos (Cabero y Barroso, 2013). Particularmente, en nuestro caso, el coeficiente $\mathrm{K}$ era superior a .8 en 12 de los 20 expertos seleccionados inicialmente, por lo que fueron 12 los que finalmente nos ayudaron a validar el cuestionario. Para ello, los expertos contaban con una escala de valoración de construcción tipo Likert que confeccionamos, con una valoración de 1-4 (1 [Inadecuado] y 4 [Muy adecuado]), y con la cual pretendiamos recoger información sobre la semántica, el número, el orden o la tipología de las preguntas.

Posteriormente, procedimos a la fiabilidad del cuestionario. Como señalan Cabero, Fernández y Córdoba (2016), para alcanzar dicha fiabilidad existen diferen-

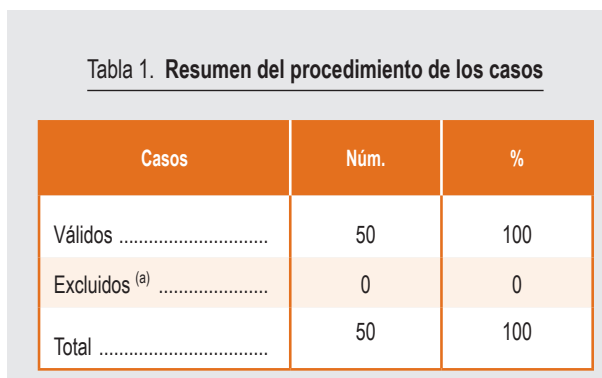

(a) La eliminación por lista se basa en todas las variables del procedimiento.

Fuente: elaboración propia.

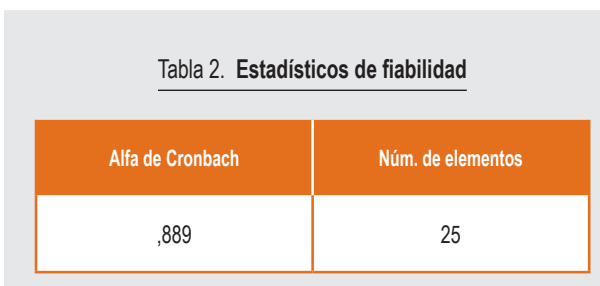

Fuente: elaboración propia tes procedimientos: medida de estabilidad, método de formas alternativas o paralelas, método de mitades partidas, coeficiente alfa de Cronbrach y coeficiente KR-20. Los dos primeros métodos requieren aplicar el cuestionario dos o más veces a un mismo grupo de personas, el quinto método es para opciones dicotómicas y el tercero y cuarto método se utilizan para escala Likert, siendo la de mayor usabilidad (Barroso y Cabero, 2010) la de Cronbrach, puesto que es la que tiene mayor potencia y flexibilidad en su aplicación (O'Dwyer y Bernauer, 2014). Con la finalidad de determinar la consistencia interna del instrumento utilizado en nuestro estudio, se aplicó la prueba de fiabilidad alfa de Cronbrach. Los resultados obtenidos se muestran en las tablas 1,2 y 3.

Si examinamos tanto el estadístico de fiabilidad como los estadísticos total-elemento de las tablas precedentes, podemos observar que las puntuaciones obtenidas fueron significativas. Concretamente, el alfa de Cronbach alcanzado en este estudio fue de 0,889 , por lo que denota el nivel de fiabilidad del instrumento, basándonos en el criterio de George y Mallery (2003) y Mateo (2004).

\begin{tabular}{|c|c|c|c|}
\multicolumn{4}{|c}{ Tabla 3. Estadísticos total-elemento } \\
\cline { 2 - 3 } Variable & $\begin{array}{c}\text { Alfa de } \\
\text { Cronbach si } \\
\text { se elimina el } \\
\text { elemento }\end{array}$ & Variable & $\begin{array}{c}\text { Alfa de } \\
\text { Cronbach si } \\
\text { se elimina el } \\
\text { elemento }\end{array}$ \\
\cline { 1 - 2 } V7 &, 880 & V10.10 &, 876 \\
V8 &, 886 & V10.11 &, 882 \\
V9 &, 892 & V10.12 &, 886 \\
V10 &, 884 & V10.13 &, 878 \\
V10.1 &, 880 & V11 &, 897 \\
V10.2 &, 878 & V11.1 &, 887 \\
\cline { 1 - 2 } V10.3 &, 878 & V11.2 &, 889 \\
V10.4 &, 877 & V11.3 &, 887 \\
V10.5 &, 879 & V11.4 &, 897 \\
V10.6 &, 878 & V11.5 &, 895 \\
V10.7 &, 877 & V11.6 &, 897 \\
V10.8 &, 875 & V12 &, 895 \\
V10.9 &, 879 & - & - \\
\hline
\end{tabular}




\section{RESULTADOS}

En el presente apartado abordaremos los principales resultados alcanzados tras el proceso de análisis de los datos obtenidos mediante el instrumento utilizado de índole cuantitativo.

Si analizamos la pertenencia a las redes sociales en función del género, los datos recogidos en la tabla 4 ponen de manifiesto que, en ambos casos, tanto en el de los hombres como en el de las mujeres, es mayor el número de sujetos que forman parte de alguna red social (en el caso de los hombres [ $f=60 ; 56,6 \%$ ] $y$ en el caso de las mujeres $[f=99 ; 52,7 \%]$ ) que los que no forman parte. Sin embargo, si comparamos el número total de hombres y el porcentaje de ellos que aseguran formar parte de la red social con el núme-

\section{[...] aunque con una inapreciable diferencia del $3,9 \%$, los datos demuestran que es mayor el porcentaje de hombres que pertenecen a una red social}

ro total de mujeres y el porcentaje de ellas que dicen formar parte de la red social, aunque con una inapreciable diferencia del $3,9 \%$, los datos demuestran que es mayor el porcentaje de hombres que pertenecen a una red social.

\begin{tabular}{|c|c|c|c|c|c|c|}
\hline & \multicolumn{6}{|c|}{ ¿Pertenece a alguna red social? } \\
\hline & \multicolumn{2}{|c|}{ Si } & \multicolumn{2}{|c|}{ № } & \multicolumn{2}{|c|}{ Total } \\
\hline & $f$ & $\%$ & $f$ & $\%$ & $f$ & $\%$ \\
\hline Hombre & 60 & 56,6 & 46 & 43,4 & 106 & 100 \\
\hline 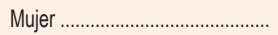 & 99 & 52,7 & 89 & 47,3 & 188 & 100 \\
\hline
\end{tabular}

En relación con el género y el nivel de interés por las redes sociales, en la tabla 5 y en la figura 1 se puede apreciar que, en el caso de los hombres, la frecuencia más elevada la encontramos bajo la opción «Bastante» ( $f=48 ; 45,3 \%$ ), mientras que, en el caso de las mujeres, su mayor frecuencia se alcanza bajo la opción «Poco» ( $f=78 ; 41,49 \%)$. En ambos sexos, el índice de menor frecuencia se da bajo la opción «Mucho». En conclusión, es mayor el interés existente entre los hombres que entre las mujeres.

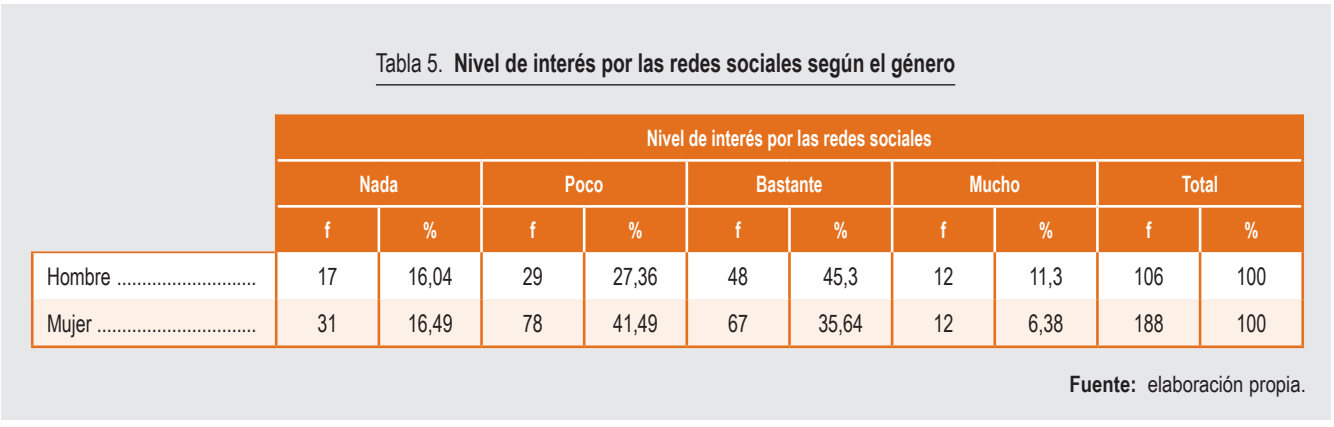




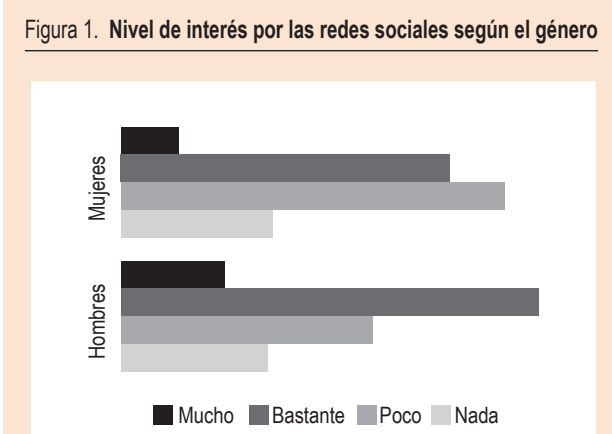

Fuente: elaboración propia.

Si analizamos la asociación existente entre la variable «Grado en que puede favorecer el uso de las redes sociales» y la variable "Género», podemos observar, en la tabla 6 , que si agrupamos las frecuencias bajo la opción «Bastante» y «Mucho», en el caso de los hombres, el índice más elevado en el uso de dichas herramientas lo hallamos en la opción «Permiten estar en contacto con amigos y familiares» ( $f=90 ; 84,9 \%$ ), seguido de la opción «Para buscar información» ( $f=$ $=87 ; 82,1 \%) y$, en tercer lugar, de la opción «Permiten conocer y manejar las nuevas tecnologías» ( $f=$ $=80 ; 75,4 \%$ ). Si nos centramos en las mujeres, el índice más elevado corresponde, en primer lugar, a la opción «Para buscar información» ( $f=160 ; 85,1 \%$ ), seguido de la opción «Permiten estar en contacto con amigos $y$ familiares» ( $f=152 ; 80,8 \%$ ) y, en tercer lugar, la opción «Permiten ampliar nuestro conocimiento» $(f=141 ; 75 \%)$. Si nos fijamos en los menores porcentajes obtenidos en el caso de los hombres, observamos que estos consideran que los aspectos que menos favorecen su uso de las redes sociales son la opción «Para conocer gente nueva» ( $f=45 ; 42,6 \%$ ), seguido de la opción «Favorecen las relaciones intergeneracionales» ( $f=51 ; 48,1 \%$ ), mientras que, en el caso de las mujeres, estas estiman que los aspectos que menos favorecen su uso son la opción «Favorecen el desarrollo personal» ( $f=41 ; 21,8 \%$ ), seguido de la opción «Para conocer gente nueva» $(f=69 ; 36,7 \%$ ).

\begin{tabular}{|c|c|c|c|c|c|c|c|c|c|}
\hline \multirow{2}{*}{\multicolumn{2}{|c|}{ Grado en que puede favorecer el uso de las redes sociales }} & \multicolumn{2}{|c|}{ Nada } & \multicolumn{2}{|c|}{ Poco } & \multicolumn{2}{|c|}{ Bastante } & \multicolumn{2}{|c|}{ Mucho } \\
\hline & & f & $\%$ & $f$ & $\%$ & $f$ & $\%$ & f & $\%$ \\
\hline \multirow{2}{*}{ Para buscar información } & Hombre & 5 & 4,7 & 14 & 13,2 & 38 & 35,9 & 49 & 46,2 \\
\hline & Mujer & 18 & 9,6 & 10 & 5,3 & 74 & 39,4 & 86 & 45,7 \\
\hline \multirow{2}{*}{$\begin{array}{l}\text { Favorecen las relaciones interge- } \\
\text { neracionales }\end{array}$} & Hombre & 17 & 16 & 38 & 3,9 & 33 & 31,1 & 18 & 17 \\
\hline & Mujer & 62 & 33 & 37 & 19,7 & 62 & 33 & 27 & 14,4 \\
\hline \multirow{2}{*}{$\begin{array}{l}\text { Integración de las personas mayo- } \\
\text { res en la sociedad }\end{array}$} & Hombre & 12 & 11,3 & 25 & 23,6 & 48 & 45,3 & 21 & 19,8 \\
\hline & Mujer & 40 & 21,3 & 48 & 25,5 & 66 & 53,1 & 34 & 18,1 \\
\hline \multirow{2}{*}{$\begin{array}{l}\text { Permiten o fomentan las relaciones } \\
\text { sociales }\end{array}$} & Hombre & 12 & 11,3 & 29 & 27,4 & 34 & 32,1 & 31 & 29,2 \\
\hline & & 35 & 18,6 & 36 & 19,1 & 79 & 42 & 38 & 20,2 \\
\hline \multirow{2}{*}{$\begin{array}{l}\text { Compartir conocimiento, fotos, ví- } \\
\text { deos, enlaces, etc. }\end{array}$} & Hombre & 11 & 10,4 & 21 & 19,8 & 47 & 44,3 & 27 & 25,5 \\
\hline & Mujer & 26 & 13,8 & 18 & 9,6 & 71 & 37,8 & 73 & 38,8 \\
\hline
\end{tabular}




\begin{tabular}{|c|c|c|c|c|c|c|c|c|c|}
\hline \multicolumn{10}{|c|}{ Tabla 6. Grado de utilidad de las redes sociales en función del género (cont.) } \\
\hline \multirow{2}{*}{\multicolumn{2}{|c|}{ Grado en que puede favorecer el uso de las redes sociales }} & \multicolumn{2}{|c|}{ Nada } & \multicolumn{2}{|c|}{ Poco } & \multicolumn{2}{|c|}{ Bastante } & \multicolumn{2}{|c|}{ Mucho } \\
\hline & & $f$ & $\%$ & $f$ & $\%$ & $f$ & $\%$ & $f$ & $\%$ \\
\hline \multicolumn{10}{|l|}{$\ldots / \ldots$} \\
\hline $\begin{array}{l}\text { Permiten estar en contacto con } \\
\text { amigos y familiares }\end{array}$ & $\begin{array}{l}\text { Hombre } \\
\text { Mujer }\end{array}$ & $\begin{array}{l}6 \\
21\end{array}$ & $\begin{array}{l}5,7 \\
11,2\end{array}$ & $\begin{array}{l}10 \\
15\end{array}$ & $\begin{array}{c}9,4 \\
8\end{array}$ & $\begin{array}{l}50 \\
66\end{array}$ & $\begin{array}{l}47,2 \\
35,1\end{array}$ & $\begin{array}{l}40 \\
86\end{array}$ & $\begin{array}{l}37,7 \\
45,7\end{array}$ \\
\hline Favorecen el desarrollo personal & $\begin{array}{l}\text { Hombre } \\
\text { Mujer }\end{array}$ & $\begin{array}{l}16 \\
42\end{array}$ & $\begin{array}{l}15,1 \\
22,3\end{array}$ & $\begin{array}{l}34 \\
45\end{array}$ & $\begin{array}{l}32,1 \\
23,9\end{array}$ & 42 & $\begin{array}{l}39,6 \\
1,6\end{array}$ & $\begin{array}{l}14 \\
38\end{array}$ & $\begin{array}{l}13,2 \\
20,2\end{array}$ \\
\hline $\begin{array}{l}\text { Permiten ampliar nuestro conoci- } \\
\text { miento }\end{array}$ & $\begin{array}{l}\text { Hombre } \\
\text { Mujer }\end{array}$ & $\begin{array}{l}7 \\
31\end{array}$ & $\begin{array}{l}6,6 \\
16,5\end{array}$ & $\begin{array}{l}20 \\
16\end{array}$ & $\begin{array}{l}18,9 \\
8,5\end{array}$ & $\begin{array}{l}46 \\
76\end{array}$ & $\begin{array}{l}43,4 \\
40,4\end{array}$ & $\begin{array}{l}33 \\
65\end{array}$ & $\begin{array}{l}31,1 \\
34,6\end{array}$ \\
\hline $\begin{array}{l}\text { Permiten conocer y manejar las } \\
\text { nuevas tecnologías }\end{array}$ & $\begin{array}{l}\text { Hombre } \\
\text { Mujer }\end{array}$ & $\begin{array}{l}7 \\
30\end{array}$ & $\begin{array}{l}6,6 \\
16\end{array}$ & $\begin{array}{l}19 \\
30\end{array}$ & $\begin{array}{c}17,9 \\
16\end{array}$ & $\begin{array}{l}47 \\
73\end{array}$ & $\begin{array}{l}44,3 \\
38,8\end{array}$ & $\begin{array}{l}33 \\
55\end{array}$ & $\begin{array}{l}31,1 \\
29,2\end{array}$ \\
\hline Permiten entretenerte y divertirte & $\begin{array}{l}\text { Hombre } \\
\text { Mujer }\end{array}$ & $\begin{array}{l}12 \\
45\end{array}$ & $\begin{array}{l}11,3 \\
23,9\end{array}$ & $\begin{array}{l}18 \\
41\end{array}$ & $\begin{array}{c}17 \\
21,8\end{array}$ & $\begin{array}{l}54 \\
61\end{array}$ & $\begin{array}{l}50,9 \\
32,4\end{array}$ & $\begin{array}{l}22 \\
41\end{array}$ & $\begin{array}{l}20,8 \\
21,8\end{array}$ \\
\hline $\begin{array}{l}\text { Favorecen la autonomía de los } \\
\text { mayores }\end{array}$ & $\begin{array}{l}\text { Hombre } \\
\text { Mujer }\end{array}$ & $\begin{array}{l}13 \\
36\end{array}$ & $\begin{array}{l}12,3 \\
19,1\end{array}$ & $\begin{array}{l}31 \\
46\end{array}$ & $\begin{array}{l}29,2 \\
24,5\end{array}$ & $\begin{array}{l}46 \\
73\end{array}$ & $\begin{array}{l}24,5 \\
38,8\end{array}$ & $\begin{array}{l}16 \\
31\end{array}$ & $\begin{array}{l}15,1 \\
16,5\end{array}$ \\
\hline Para conocer gente nueva & $\begin{array}{l}\text { Hombre } \\
\text { Mujer }\end{array}$ & $\begin{array}{l}28 \\
66\end{array}$ & $\begin{array}{l}26,4 \\
35,1\end{array}$ & $\begin{array}{l}33 \\
53\end{array}$ & $\begin{array}{l}31,1 \\
28,2\end{array}$ & $\begin{array}{l}23 \\
48\end{array}$ & $\begin{array}{c}22 \\
25,5\end{array}$ & $\begin{array}{l}22 \\
21\end{array}$ & $\begin{array}{l}20,6 \\
11,2\end{array}$ \\
\hline $\begin{array}{l}\text { Favorecen el desarrollo mental/ } \\
\text { intelectual }\end{array}$ & $\begin{array}{l}\text { Hombre } \\
\text { Mujer }\end{array}$ & $\begin{array}{l}11 \\
31\end{array}$ & $\begin{array}{l}10,4 \\
16,5\end{array}$ & $\begin{array}{l}29 \\
31\end{array}$ & $\begin{array}{l}27,4 \\
16,5\end{array}$ & $\begin{array}{l}46 \\
83\end{array}$ & $\begin{array}{l}43,4 \\
44,1\end{array}$ & $\begin{array}{l}20 \\
43\end{array}$ & $\begin{array}{l}18,9 \\
22,9\end{array}$ \\
\hline $\begin{array}{l}\text { Para adquirir nuevas habilidades/ } \\
\text { capacidades }\end{array}$ & $\begin{array}{l}\text { Hombre } \\
\text { Mujer }\end{array}$ & $\begin{array}{l}13 \\
38\end{array}$ & $\begin{array}{l}12,3 \\
20,2\end{array}$ & $\begin{array}{l}25 \\
35\end{array}$ & $\begin{array}{l}23,6 \\
18,6\end{array}$ & $\begin{array}{l}53 \\
80\end{array}$ & $\begin{array}{c}50 \\
42,6\end{array}$ & $\begin{array}{l}15 \\
35\end{array}$ & $\begin{array}{l}14,2 \\
18,6\end{array}$ \\
\hline
\end{tabular}

Por otro lado, si indagamos en la asociación existente entre la variable "Género», pero esta vez asociada a la variable "Grado en que puede perjudicar el uso de las redes sociales», podemos observar, en la tabla 7 , que si agrupamos las frecuencias bajo la opción «Bastante» y «Mucho», el índice de aparición más elevado, tanto en el caso de las mujeres $(f=143 ; 76,1 \%)$, como en el caso de los hombres ( $f=86 ; 81,2 \%)$, lo hallamos bajo la opción «Desconfianza. Existen perfiles falsos». Sí encontramos diferencias en cuanto a la segunda y tercera opción considerada como más peligrosa respecto al uso de las redes sociales entre hombres y mujeres. En el caso de los hombres, estos exponen como segundo aspecto más perjudicial el «Acceso a contenidos inadecuados» ( $f=80 ; 75,4 \%$ ), seguido de las opciones «Pérdida de privacidad» $\mathrm{e}$ «Inseguridad, robos, fraudes, etc.», ambas con ( $f=79 ; 74,5 \%$ ). En el caso de las mujeres, el segundo aspecto considerado como más perjudicial en relación con el uso de las redes sociales son las opciones «Pérdida de interacción cara a cara» e «Inseguridad, robos, fraudes, etc.», ambas con ( $f=141 ; 75 \%$ ), y, en tercer lugar, la opción «Pérdida de privacidad», que conlleva a veces su uso ( $f=136$; $72,3 \%$ ). Tanto hombres como mujeres consideran que la opción «Pérdida de tiempo» es una de las variables menos perjudiciales. 
Uso e interés por las redes sociales entre las personas mayores desde una perspectiva de género. / J. Barroso y S. Aguilar Un estudio en las universidades de mayores andaluzas

\begin{tabular}{|c|c|c|c|c|c|c|c|c|c|}
\hline \multirow{2}{*}{\multicolumn{2}{|c|}{$\begin{array}{l}\text { Grado en que puede perjudicar } \\
\text { el uso de las redes sociales }\end{array}$}} & \multicolumn{2}{|c|}{ Nada } & \multicolumn{2}{|c|}{ Poco } & \multicolumn{2}{|c|}{ Bastante } & \multicolumn{2}{|c|}{ Mucho } \\
\hline & & f & $\%$ & $f$ & $\%$ & $f$ & $\%$ & f & $\%$ \\
\hline \multirow{2}{*}{ Pérdida de privacidad } & Hombre & 9 & 8,5 & 18 & 16,9 & 37 & 34,9 & 42 & 39,6 \\
\hline & Mujer & 18 & 9,6 & 34 & 18,1 & 56 & 29,8 & 80 & 42,5 \\
\hline \multirow{2}{*}{ Adicción } & Hombre & 11 & 10,4 & 19 & 17,9 & 39 & 36,8 & 37 & 34,9 \\
\hline & Mujer & 22 & 11,7 & 31 & 16,5 & 48 & 25,5 & 87 & 46,3 \\
\hline \multirow{2}{*}{ Inseguridad, robos, fraudes, etc. } & Hombre & 10 & 9,4 & 17 & 16 & 33 & 31,1 & 46 & 43,4 \\
\hline & Mujer & 20 & 10,6 & 27 & 14,4 & 65 & 34,6 & 76 & 40,4 \\
\hline \multirow{2}{*}{ Acceso a contenidos inadecuados } & Hombre & 11 & 10,4 & 15 & 14,2 & 42 & 39,6 & 38 & 35,8 \\
\hline & Mujer & 29 & 15,4 & 31 & 16,5 & 52 & 27,7 & 76 & 40,4 \\
\hline \multirow{2}{*}{ Pérdida de tiempo } & Hombre & 28 & 26,4 & 33 & 31,1 & 31 & 29,2 & 14 & 13,2 \\
\hline & Mujer & 51 & 27,1 & 57 & 30,3 & 36 & 19,1 & 44 & 23,4 \\
\hline \multirow{2}{*}{ Desconfianza. Existen perfiles falsos } & Hombre & 7 & 6,6 & 13 & 12,3 & 45 & 42,5 & 41 & 38,7 \\
\hline & Mujer & 16 & 8,5 & 29 & 15,4 & 53 & 28,2 & 90 & 47,9 \\
\hline \multirow{2}{*}{ Pérdida de interacción cara a cara } & Hombre & 14 & 13,2 & 18 & 17 & 27 & 25,5 & 47 & 44,3 \\
\hline & Mujer & 29 & 15,4 & 18 & 9,6 & 45 & 23,9 & 96 & 51,1 \\
\hline
\end{tabular}

Fuente: elaboración propia.

Si estudiamos la frecuencia de acceso a las redes sociales, encontramos diferencias significativas (véase tabla 8). En el caso concreto de los hombres, la mayor frecuencia se obtiene bajo la opción «A diario» ( $f=30 ; 50 \%$ ), mientras que en el caso de las mujeres su mayor frecuencia se alcanza bajo la opción « 203 veces por semana» ( $f=43 ; 43,4 \%$ ), por lo que los datos nos revelan que los hombres se conectan con mayor frecuencia que las mujeres. En ambos casos, el número de sujetos que exponen conectarse con poca frecuencia bajo la opción «1 vez por semana», «Al menos 1 vez cada 15 días» y «De 1 a 3 veces al mes» es escaso. Dicha asociación queda representada en la figura 2.

\begin{tabular}{|c|c|c|c|c|c|c|c|c|c|c|c|c|}
\hline & & & \multicolumn{10}{|c|}{ Tabla 8. Género y frecuencia de acceso a las redes sociales } \\
\hline & \multicolumn{12}{|c|}{ ¿Cada cuánto tiempo consultas las redes sociales? } \\
\hline & \multicolumn{2}{|c|}{ A diario } & \multicolumn{2}{|c|}{$\begin{array}{l}203 \text { veces } \\
\text { por semana }\end{array}$} & \multicolumn{2}{|c|}{1 vez por semana } & \multicolumn{2}{|c|}{$\begin{array}{l}\text { Al menos } 1 \text { vez } \\
\text { cada } 15 \text { dias }\end{array}$} & \multicolumn{2}{|c|}{ De 1 a 3 veces al mes } & \multicolumn{2}{|c|}{ Total } \\
\hline & f & $\%$ & f & $\%$ & f & $\%$ & f & $\%$ & $f$ & $\%$ & $f$ & $\%$ \\
\hline Hombre .... & 30 & 50 & 21 & 35 & 4 & 6,7 & 3 & 5 & 2 & 3,3 & 60 & 100 \\
\hline Mujer ......... & 36 & 36,4 & 43 & 43,4 & 17 & 17,2 & 2 & 2 & 1 & 1 & 99 & 100 \\
\hline
\end{tabular}

Fuente: elaboración propia. 
Figura 2. Género y frecuencia de acceso a las redes sociales

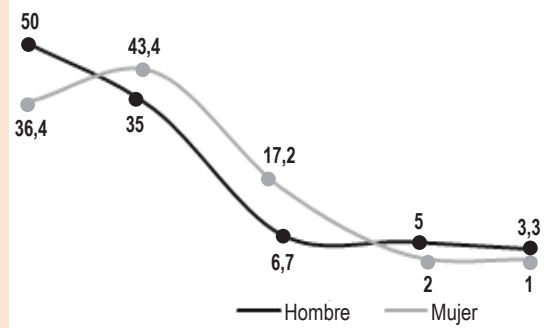

Fuente: elaboración propia.

Tabla 9. Contingencia: género y factores motivacionales de uso

\begin{tabular}{|l|c|c|c|c|c|}
\multicolumn{2}{|c|}{$\begin{array}{l}\text { Motivo por el que utiliza las } \\
\text { redes sociales }\end{array}$} & \multicolumn{2}{|c|}{ Si } & \multicolumn{2}{c}{ No } \\
\cline { 2 - 6 } & $f$ & $\%$ & $f$ & $\%$ \\
\hline $\begin{array}{l}\text { La utilizan mis } \\
\text { amigos }\end{array}$ & Hombre & 38 & 35,8 & 22 & 20,7 \\
Es fácil de usar & Mujer & 69 & 36,7 & 30 & 15,9 \\
\hline Es la única que & Hombre & 38 & 35,8 & 22 & 20,7 \\
conozco & Mujer & 70 & 37,2 & 29 & 15,4 \\
\hline Es atractiva & Mujer & 20 & 10,6 & 79 & 42 \\
\hline Es la que mejor & Hombre & 6 & 5,7 & 54 & 50,9 \\
sé manejar & Mujer & 17 & 9 & 82 & 43,6 \\
\hline Es rápida & Mujer & 20 & 18,9 & 40 & 37,7 \\
\hline & Hombre & 14 & 19,1 & 63 & 33,5 \\
\hline & Mujer & 21 & 11,2 & 78 & 41,5 \\
\hline
\end{tabular}

Fuente: elaboración propia.
Con el fin de conocer si existían diferencias motivacionales a la hora de formar parte de las herramientas sociales entre los diferentes géneros, estudiamos la asociación entre ambas variables. Como podemos apreciar en la tabla 9, el principal motivo por el que los hombres utilizan estas herramientas está vinculado a las opciones "La utilizan mis amigos» y «Es fácil de usar», ambas con ( $f=38 ; 35,8 \%$ ), seguido de la opción «Es la que mejor sé manejar» $(\mathrm{f}=20 ; 18,9 \%) \mathrm{y}$, en tercer lugar, de la opción «Es rápida» ( $f=14 ; 13,2 \%)$. En el caso de las mujeres, seleccionan como primera opción «Es fácil de usar» ( $f=70 ; 37,2 \%)$, en segunda posición «La utilizan mis amigos» ( $f=69 ; 36,7 \%)$ y, finalmente, en tercer lugar, «Es la que mejor sé manejar» ( $f=36 ; 19,1 \%$ ). En cuanto a las menores frecuencias y porcentajes obtenidos, ambos géneros coinciden en que la opción «Es segura» no es uno de los principales motivos por los que utilizan las redes sociales.

A continuación expondremos algunas de las finalidades con las que son utilizadas las herramientas por las personas mayores, incidiendo en las diferencias que se producen entre hombres y mujeres. Si observamos detenidamente las frecuencias y los porcentajes obtenidos para cada uno de los aspectos a valorar por los participantes en la tabla 10, hallamos que, en el caso de los hombres, su principal finalidad cuando se conectan a la red social se centra en la opción «Para obtener información que cuelgan mis contactos» ( $f=$ $=31 ; 19,5 \%$ ), seguido de las opciones «Para estar en contacto con mis amigos» ( $f=30 ; 18,9 \%)$ e «Informarme de noticias de actualidad» ( $f=29 ; 18,2 \%$ ). Con escasa diferencia le sigue la opción «Aprender cosas nuevas» ( $f=28 ; 17,6 \%$ ) y, por último, «Compartir/subir fotos, vídeos, música, etc.» ( $f=26 ; 16,3 \%)$. En el caso de las mujeres, su principal objetivo es la opción «Para estar en contacto con mis amigos» ( $f=56 ; 35,2 \%$ ), seguido de las opciones «Informarme de noticias de actualidad» ( $f=55 ; 34,6 \%$ ), «Aprender cosas nuevas» ( $f=51 ; 32,1 \%$ ), «Compartir/subir fotos, vídeos, música, etc.» ( $\mathrm{f}=45 ; 28,3 \%$ ) y, en última posición, y a diferencia de los hombres, que lo exponían como primera opción, «Para obtener información que cuelgan mis contactos» ( $f=42 ; 26,4 \%$ ). Si nos centramos en averiguar para qué no suelen utilizar las redes sociales, en el caso de los hombres las opciones seleccionadas son «Para jugar», «Para buscar pareja» 0 «Para hacer amigos nuevos», todas con una misma frecuencia ( $f=$ $=59 ; 37,1 \%$ ), datos que coinciden con los ofrecidos por las mujeres, que igualmente manifiestan no utili- 


\begin{tabular}{|c|c|c|c|c|c|}
\hline \multicolumn{6}{|c|}{$\begin{array}{l}\text { Tabla 10. Contingencia: género y fin con el que se utiliza la } \\
\text { red social }\end{array}$} \\
\hline \multirow{2}{*}{\multicolumn{2}{|c|}{$\begin{array}{l}\text { Fin con el que utilizan } \\
\text { las redes sociales }\end{array}$}} & \multicolumn{2}{|c|}{$\mathrm{Si}$} & \multicolumn{2}{|c|}{ No } \\
\hline & & $f$ & $\%$ & $f$ & $\%$ \\
\hline \multirow{2}{*}{ Para chatear } & Hombre & 12 & 7,5 & 48 & 30,2 \\
\hline & Mujer & 7 & 4,4 & 92 & 57,9 \\
\hline \multirow{2}{*}{ Para jugar } & Hombre & 4 & 2,5 & 59 & 37,1 \\
\hline & Mujer & 9 & 5,6 & 90 & 56,6 \\
\hline \multirow{2}{*}{$\begin{array}{l}\text { Aprender cosas } \\
\text { nuevas }\end{array}$} & Hombre & 28 & 17,6 & 32 & 20,1 \\
\hline & Mujer & 51 & 32,1 & 48 & 30,2 \\
\hline \multirow{2}{*}{$\begin{array}{l}\text { Compartir/subir fotos, } \\
\text { videos, música, etc. }\end{array}$} & Hombre & 26 & 16,3 & 34 & 21,4 \\
\hline & Mujer & 45 & 28,3 & 54 & 34 \\
\hline \multirow{2}{*}{$\begin{array}{l}\text { Para publicar infor- } \\
\text { mación de estudio o } \\
\text { temas afines }\end{array}$} & Hombre & 11 & 6,9 & 49 & 30,8 \\
\hline & Mujer & 9 & 5,7 & 90 & 56,6 \\
\hline \multirow{2}{*}{$\begin{array}{l}\text { Para obtener infor- } \\
\text { mación que cuelgan } \\
\text { mis contactos }\end{array}$} & Hombre & 31 & 19,5 & 29 & 18,2 \\
\hline & Mujer & 42 & 26,4 & 57 & 35,8 \\
\hline \multirow{2}{*}{ Para buscar pareja } & Hombre & 1 & 0,6 & 59 & 37,1 \\
\hline & Mujer & 1 & 0,6 & 98 & 61,6 \\
\hline \multirow{2}{*}{$\begin{array}{l}\text { Para hacer amigos } \\
\text { nuevos }\end{array}$} & Hombre & 1 & 0,6 & 59 & 37,1 \\
\hline & Mujer & 2 & 1,3 & 97 & 61 \\
\hline \multirow{2}{*}{$\begin{array}{l}\text { Informarme de } \\
\text { noticias de actualidad }\end{array}$} & Hombre & 29 & 18,2 & 31 & 19,5 \\
\hline & Mujer & 55 & 34,6 & 44 & 27,7 \\
\hline \multirow{2}{*}{$\begin{array}{l}\text { Para estar en contac- } \\
\text { to con mis amigos }\end{array}$} & Hombre & 30 & 18,9 & 30 & 18,9 \\
\hline & Mujer & 56 & 35,2 & 43 & 27 \\
\hline \multirow{2}{*}{$\begin{array}{l}\text { Enviar mensajes } \\
\text { públicos }\end{array}$} & Hombre & 5 & 3,1 & 55 & 34,6 \\
\hline & Mujer & 11 & 6,9 & 88 & 55,3 \\
\hline \multirow{2}{*}{$\begin{array}{l}\text { Enviar mensajes } \\
\text { privados }\end{array}$} & Hombre & 15 & 9,4 & 45 & 28,3 \\
\hline & Mujer & 37 & 23,3 & 62 & 39 \\
\hline
\end{tabular}

zar las redes sociales «Para buscar pareja» ( $f=98$; $61,6 \%$ ) ni «Para hacer amigos nuevos» ( $f=97 ; 61 \%$ ).

Asimismo, consideramos interesante analizar esta misma variable, «Fin con el que utiliza las redes sociales», pero asociado a la variable "Estado civil», para conocer si existen diferencias significativas. Si observamos la tabla 11 , las frecuencias y los porcentajes derivados de tal asociación nos dejan ver que no existen grandes diferencias entre el estado civil del sujeto y el objetivo con el que utiliza la herramienta. Concretamente, tanto en el caso de los/as casados/as ( $f=51 ; 32,1 \%$ ), como de los/as solteros/as $(f=9 ; 5,7 \%$ ), la opción que obtiene mayor frecuencia es «Informarme de noticias de actualidad». Sí existen diferencias con respecto a su segunda opción, siendo para los casados «Aprender cosas nuevas» ( $f=50 ; 31,4 \%$ y para los solteros «Para estar en contacto con mis amigos» ( $f=7 ; 4,4 \%)$. En el caso de los/as divorciados/as $(f=10 ; 6,3 \%)$ y viudos/as $(f=$ $=18 ; 11,3 \%)$, su primer fin, según los datos, se centra en la opción «Para estar en contacto con mis amigos»; en segundo lugar, los/las divorciados/as $(f=8 ; 5 \%$ ) y los/as viudos/as $(f=17 ; 10,7 \%)$ coinciden bajo la opción «Para compartir/subir fotos, vídeos, música, etc.». Hay que destacar que, mientras que para los/as divorciados/ as, entre su segunda opción también destaca «Para obtener información que cuelgan mis contactos» ( $f=8$; $5 \%$ ), para los/as viudos/as ( $f=14 ; 8,8 \%$ ) esta sería su tercera opción. En tercer lugar, los/as divorciados/as ( $\mathrm{f}=$ $=7 ; 4,4 \%$ ) exponen utilizarla para «Informarme de noticias de actualidad», opción principal que era, como hemos comentado anteriormente, para casados/as y solteros/as. En el caso de los/as viudos/as, su tercera opción sería «Aprender cosas nuevas» ( $f=15 ; 9,4 \%$ ).

Por último, y teniendo en cuenta los cuatro estados civiles, hay que señalar que tanto casados/as, como solteros/as y divorciados/as no utilizan la opción «Para buscar pareja» ni la opción «Hacer amigos nuevos».

En relación con las herramientas utilizadas, si sumamos las opciones «Bastante» y «Mucho», tal y como podemos apreciar en la tabla 12, tanto los hombres como las mujeres participantes en este estudio dicen ser usuarios con mayor frecuencia y en el mismo orden de secuencia de Facebook, YouTube y Messenger, por lo que existe concordancia de acuerdo con la frecuencia de aparición de los indicadores entre ambos sexos. En el caso de los hombres, destacan «Facebook» ( $\mathrm{f}=23 ; 38,3 \%)$, «YouTube» $(f=24 ; 40 \%)$ y «Messenger» $(f=20 ; 33,3 \%)$. En el caso 


\begin{tabular}{|c|c|c|c|c|c|}
\hline \multirow{2}{*}{\multicolumn{2}{|c|}{ Fin con el que utiliza la red }} & \multicolumn{2}{|c|}{$\mathrm{Si}$} & \multicolumn{2}{|c|}{ No } \\
\hline & & f & $\%$ & f & $\%$ \\
\hline \multirow{4}{*}{ Para chatear } & Casado/a & 9 & 5,7 & 90 & 56,6 \\
\hline & Soltero/a & 1 & 0,6 & 13 & 8,2 \\
\hline & Divorciado/a & 3 & 1,9 & 14 & 8,8 \\
\hline & Viudo/a & 6 & 3,8 & 23 & 14,4 \\
\hline \multirow{4}{*}{ Para jugar } & Casado/a & 5 & 3,1 & 94 & 59,1 \\
\hline & Soltero/a & 1 & 0,6 & 13 & 8,2 \\
\hline & Divorciado/a & 2 & 1,3 & 15 & 9,4 \\
\hline & Viudo/a & 5 & 3,1 & 24 & 15,1 \\
\hline \multirow{4}{*}{$\begin{array}{l}\text { Aprender cosas } \\
\text { nuevas }\end{array}$} & Casado/a & 50 & 31,4 & 49 & 30,8 \\
\hline & Soltero/a & 6 & 3,8 & 8 & 5 \\
\hline & Divorciadola & 8 & 5 & 9 & 5,7 \\
\hline & Viudo/a & 15 & 9,4 & 14 & 8,8 \\
\hline \multirow{4}{*}{$\begin{array}{l}\text { Compartir/subir } \\
\text { fotos, videos, } \\
\text { música, etc. }\end{array}$} & Casado/a & 43 & 27 & 56 & 35,2 \\
\hline & Solterola & 3 & 1,9 & 11 & 6,9 \\
\hline & Divorciado/a & 8 & 5 & 9 & 5,7 \\
\hline & Viudo/a & 17 & 10,7 & 12 & 7,5 \\
\hline \multirow{4}{*}{$\begin{array}{l}\text { Para publicar } \\
\text { información de } \\
\text { estudio o temas } \\
\text { afines }\end{array}$} & Casado/a & 13 & 8,2 & 86 & 54,1 \\
\hline & Soltero/a & 1 & 0,6 & 13 & 8,2 \\
\hline & Divorciado/a & 1 & 0,6 & 16 & 10,1 \\
\hline & Viudo/a & 5 & 3,1 & 24 & 15,1 \\
\hline \multirow{4}{*}{$\begin{array}{l}\text { Para obtener } \\
\text { información que } \\
\text { cuelgan mis } \\
\text { contactos }\end{array}$} & Casado/a & 47 & 29,5 & 52 & 32,7 \\
\hline & Soltero/a & 4 & 2,5 & 10 & 6,3 \\
\hline & Divorciado/a & 8 & 5 & 9 & 5,7 \\
\hline & Viudo/a & 14 & 8,8 & 15 & 9,4 \\
\hline \multirow{4}{*}{ Buscar pareja } & Casado/a & - & - & 99 & 62,3 \\
\hline & Soltero/a & - & - & 14 & 8,8 \\
\hline & Divorciado/a & - & - & 17 & 10,7 \\
\hline & Viudo/a & 2 & 1,2 & 27 & 17 \\
\hline \multirow{4}{*}{$\begin{array}{l}\text { Hacer amigos } \\
\text { nuevos }\end{array}$} & Casado/a & - & - & 99 & 62,3 \\
\hline & Soltero/a & - & - & 14 & 8,8 \\
\hline & Divorciadola & - & - & 17 & 10,7 \\
\hline & Viudo/a & 3 & 1,9 & 26 & 16,4 \\
\hline
\end{tabular}

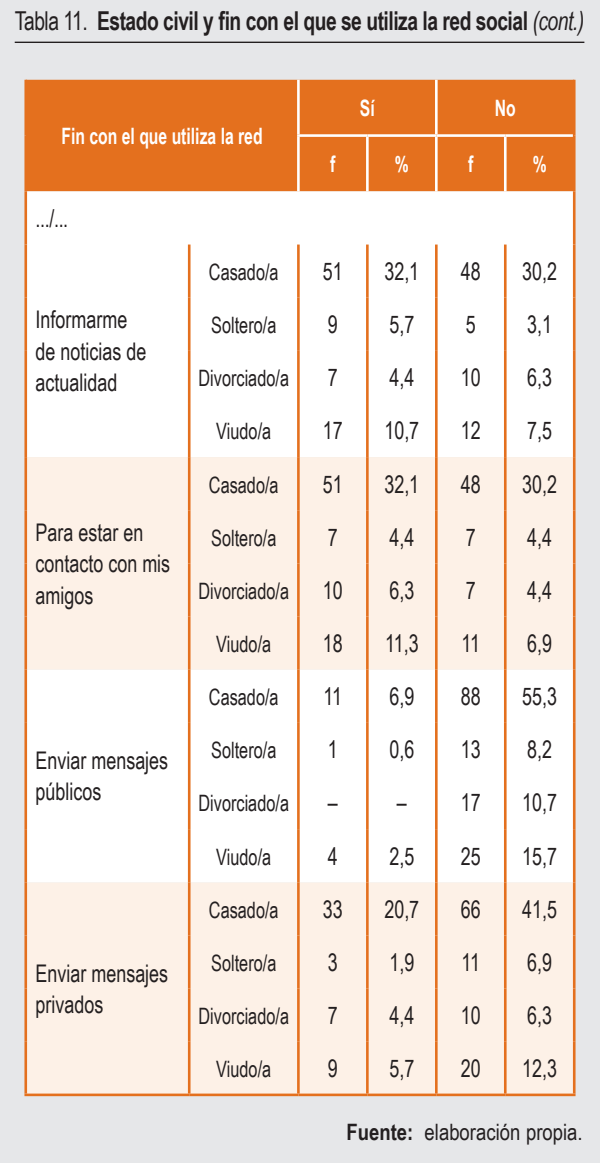

de las mujeres, los resultados han sido «Facebook» ( $f=$ 41; $41,5 \%)$, «YouTube» ( $f=29 ; 29,3 \%)$ y «Messenger» $(\mathrm{f}=27 ; 27,3 \%)$. En cuanto a las herramientas que menos utilizan ambos géneros, aunque con diferentes frecuencias alcanzadas, siguen el mismo orden. En el caso de los hombres, las herramientas a las que menos acceden bajo la opción «Nada» y «Poco» son «Edublog» ( $f=60$; $100 \%$ ), «Delicious» ( $f=59 ; 98,3 \%)$ y «Videoblog» $(f=$ 58; 96,7\%), seguido de «Blog» y «Tuenti», ambos con ( $f=55 ; 91,6 \%$ ), $y$ «Wiki», «Twitter» $y$ «Windows Live», las tres con ( $f=53 ; 88,3 \%)$. En relación con las mujeres, igualmente, son menos usuarias de «Edublog» $\mathrm{y}$ «Videoblog», ambas con ( $f=99 ; 100 \%$ ), seguido de «Delicious» ( $f=97 ; 98 \%)$, «Blog» y «Tuenti», ambas con ( $f=92$; $92,9 \%)$, «Twitter» ( $f=88 ; 88,8 \%)$, «Wiki» ( $f=87 ; 87,9 \%)$ $y$ «Windows Live» $(f=82 ; 84,7 \%)$. 
Uso e interés por las redes sociales entre las personas mayores desde una perspectiva de género. / J. Barroso y S. Aguilar Un estudio en las universidades de mayores andaluzas

\begin{tabular}{|c|c|c|c|c|c|c|c|c|c|c|c|c|c|c|c|c|}
\hline & \multicolumn{11}{|c|}{ Tabla 12. Redes sociales utilizadas en función del género } & & & & & \\
\hline & & & & & & & & & \multicolumn{8}{|c|}{ Mujer } \\
\hline & \multicolumn{2}{|c|}{ Nada } & \multicolumn{2}{|c|}{ Poco } & \multicolumn{2}{|c|}{ Bastante } & \multicolumn{2}{|c|}{ Mucho } & \multicolumn{2}{|c|}{ Nada } & \multicolumn{2}{|c|}{ Poco } & \multicolumn{2}{|c|}{ Bastante } & \multicolumn{2}{|c|}{ Mucho } \\
\hline & $\mathrm{f}$ & $\%$ & f & $\%$ & $f$ & $\%$ & f & $\%$ & f & $\%$ & f & $\%$ & f & $\%$ & f & $\%$ \\
\hline Blog ............... & 50 & 83,3 & 5 & 8,3 & - & - & 5 & 8,3 & 78 & 78,8 & 14 & 14,1 & 7 & 7,1 & - & - \\
\hline Edublog ...... & 60 & 100 & - & - & - & - & - & - & 98 & 99 & 1 & 1 & - & - & - & - \\
\hline Videoblog ... & 58 & 96,7 & - & - & 1 & 1,7 & 1 & 1,7 & 96 & 97 & 3 & 3 & 1 & 1 & 1 & 1 \\
\hline Wiki ............. & 52 & 86,6 & 1 & 1,7 & 2 & 3,3 & 5 & 8,3 & 82 & 82,8 & 5 & 5,1 & 9 & 9,1 & 3 & 3 \\
\hline Delicious .... & 59 & 98,3 & - & - & 1 & 1,7 & - & - & 96 & 97 & 1 & 1 & 1 & 1 & 1 & 1 \\
\hline Facebook. & 11 & 18,3 & 26 & 43,3 & 14 & 23,3 & 9 & 15 & 24 & 24,2 & 34 & 34,3 & 25 & 25,3 & 16 & 16,2 \\
\hline Tuenti .......... & 53 & 88,3 & 2 & 3,3 & 3 & 5 & 2 & 3,3 & 87 & 87,8 & 5 & 5,1 & 5 & 5,1 & 2 & 2 \\
\hline YouTube .... & 23 & 38,3 & 13 & 21,7 & 17 & 28,3 & 7 & 11,7 & 55 & 55,5 & 15 & 15,2 & 19 & 19,2 & 10 & 10,1 \\
\hline Twitter ......... & 42 & 70 & 11 & 18,3 & 3 & 5 & 4 & 6,7 & 85 & 85,8 & 3 & 3 & 4 & 4 & 7 & 7,1 \\
\hline Messenger .. & 30 & 50 & 10 & 16,7 & 14 & 23,3 & 6 & 10 & 58 & 58,6 & 14 & 14,1 & 19 & 19,2 & 8 & 8,1 \\
\hline Windows Live & 50 & 83,3 & 3 & 5 & 3 & 5 & 4 & 6,7 & 76 & 78,6 & 6 & 6,1 & 11 & 11,1 & 6 & 6,1 \\
\hline
\end{tabular}

Fuente: elaboración propia.

Si analizamos el dispositivo de conexión utilizado en función del género y de acuerdo con la frecuencia de aparición de los indicadores de la tabla 13, mientras que para las mujeres el dispositivo más utilizado es el portátil ( $f=79 ; 42 \%$ ), para los hombres sigue siendo el ordenador fijo 0 de sobremesa ( $f=39 ; 36,8 \%$ ). Ambos sexos utilizan en tercer lugar el teléfono móvil, aunque aún sigue siendo mayor el número de sujetos que manifiestan no utilizarlo (hombres [ $f=47 ; 44,3 \%$ ] y mujeres [ $f=71 ; 37,8 \%]$ ), frente a aquellos que afirman usarlo (hombres $[f=13 ; 12,7 \%$ y yujeres $[f=28$; $14,9 \%])$. Por último, es apreciable, tras los porcentajes obtenidos, que el colectivo no usa la PDA como dispositivo de conexión tanto en el caso de las mujeres $(f=3 ; 1,6 \%)$ como en el de los hombres $(f=2 ; 1,9 \%)$.
A continuación se puede observar la figura 3 , que nos permite visualizar los datos alcanzados con respecto a la asociación citada en la tabla 13.

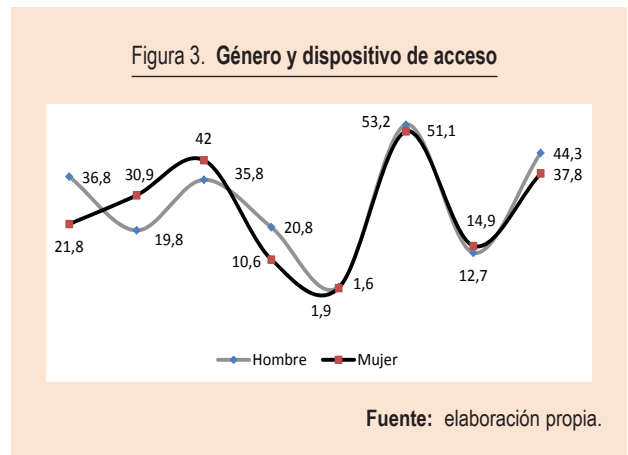

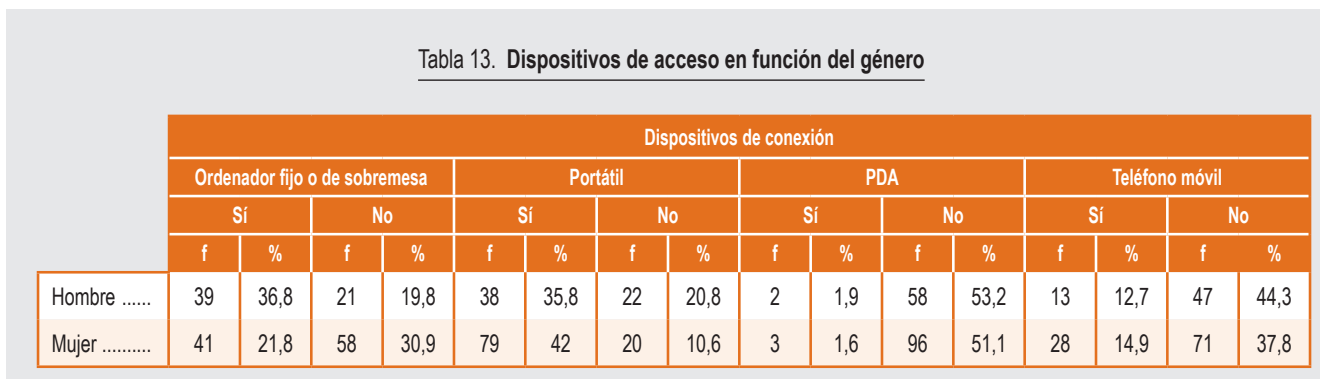

Fuente: elaboración propia. 


\section{CONCLUSIONES}

A partir de los objetivos de investigación planteados en el apartado 2.1, nos dispondremos a exponer todas las conclusiones a las que hemos podido llegar tras los datos aportados por los sujetos participantes en este estudio.

En primer lugar, no son muchas las diferencias existentes entre los hombres y las mujeres mayores con respecto a su presencia en las redes sociales, ya que en ambos casos es escasa. Sin embargo, si comparamos el número total de hombres y el porcentaje de ellos que forman parte de la red social y el número total de mujeres y el porcentaje de ellas que dicen formar parte de la red social, aunque con una inapreciable diferencia del $3,9 \%$, es mayor el porcentaje de hombres que el de mujeres. Esto nos hace ver que, a diferencia de los estudios llevados a cabo con anterioridad (INE, 2013, 2014; Abellán, A., Vilches y Pujol, 2014; Abellán y Pujol, 2015), según los cuales la brecha de género era bastante evidente, en los últimos años esta se ha ido mitigando, al igual que en el caso de los más jóvenes de nuestra población, y, actualmente, es casi inexistente.

Entre las posibles causas podemos encontrar que, a medida que pasan los años, la corresponsabilidad en el hogar, como explican Fernández y Fueyo (2014), y la formación de las mujeres (nos encontramos ante un colectivo en el que se percibe claramente un aumento del nivel de estudios, produciéndose un gran descenso del número de personas analfabetas y que, por tanto, disponen de una mayor posibilidad de acceso a las TIC, como los hombres) está cambiando, y continuará haciéndolo en los próximos años, la participación de estas en las redes sociales, no existiendo prácticamente diferencias entre los géneros con respecto a la primera brecha digital relacionada con el acceso.

Igualmente, no existen diferencias significativas entre los hombres y las mujeres relativos a los motivos que les incentivan o impulsan a formar parte de las redes sociales. Entre los principales motivos, en ambos casos, está el hecho de que sus amigos forman parte de esas redes sociales que utilizan, las consideran fáciles de usar y son las que mejor saben manejar.

\section{[...] no son muchas las} diferencias existentes entre los hombres y las mujeres mayores con respecto a su presencia en las redes sociales, ya que en ambos casos es escasa

A pesar de que se va alcanzando la igualdad de género en cuanto al acceso, sí que es cierto que, aunque ambos géneros manifiestan no tener demasiado interés por las redes sociales, este es mucho menor en las mujeres que en los hombres. La mayor preocupación entre el colectivo, coincidente en ambos géneros, es la desconfianza por la existencia de perfiles falsos en las redes sociales, por lo que el miedo se convierte en una de las barreras de inclusión de este grupo en la web social. Entre otras de sus desventajas de uso, en el caso de los hombres, está el acceso a contenidos inadecuados y la pérdida de la privacidad e inseguridad, mientras que para las mujeres son la pérdida de interacción cara a cara, la inseguridad y la adicción que conlleva a veces su uso. A diferencia de los resultados aportados por Braun (2013), donde la confianza en las redes sociales se asocia a aquellas personas mayores con un mayor interés y que ya son usuarios de las redes sociales, en nuestro caso, aunque el colectivo, tanto hombres como mujeres, forme parte de las redes sociales y tenga más o menos interés, la desconfianza sigue existiendo. Por otro lado, ambos géneros no consideran que su uso sea una pérdida de tiempo.

Con respecto a la frecuencia de acceso podemos decir que el mayor uso de las redes sociales se asocia al género masculino, que suele entrar a diario, mientras que el género femenino suelen hacerlo «2 03 veces por semana». Entre aquellos que acceden con poca frecuencia, es decir, «1 vez por semana», «Al menos 1 vez cada 15 días» $y$ «De 1 a 3 veces al mes», no existen diferencias significativas entre género. 
Son numerosas las investigaciones que han expuesto la importancia no solo del acceso a estas herramientas, sino cómo y para qué son utilizadas por los usuarios teniendo en cuenta el amplio abanico de posibilidades que ofrecen las mismas. Según los resultados de nuestro estudio, la finalidad con la que se conectan las personas mayores a las redes sociales es diferente según el género, siendo el principal motivo, para el hombre, el entretenimiento, centrado en conocer la información que cuelgan sus contactos, mientras que para la mujer esta sería su última finalidad. Dicho resultado coincide con las conclusiones ofrecidas por Colás, González y De Pablos (2013), autores que sostienen que los chicos jóvenes utilizan estas herramientas para ver lo que dicen sus amigos, las fotos que suben, etc., datos que nos hacen deducir que, en lo que respecta al uso que hacen los hombres de las redes sociales, no existen diferencias generacionales. En el caso concreto de las mujeres, su principal motivo para formar parte de las redes sociales es permanecer en contacto con sus amigos y familiares, coincidiendo con los estudios realizados por Fernández y Fueyo (2014). Sin embargo, ambos géneros coinciden como otras opciones principales de uso el informarse sobre noticias de actualidad o aprender cosas nuevas, así como no utilizarlas para buscar pareja o hacer amigos nuevos. Unas finalidades de uso que no varían en función del estado civil de las personas mayores. Independientemente de su finalidad, esto nos confirma que la actitud de ambos géneros en estas herramientas es muy diferente a la filosofía 2.0, «donde lo esencial es [...] el buen uso que se hace de ellas, pudiendo contribuir, publicar, compartir y colaborar, y evitar ser un simple espectador pasivo» (Aguilar y Benítez, 2013, pág. 7). Como nos exponían Giaroli, González y Martín (2012) tras su análisis, la mayoría no aportan opiniones personales a través de la red.

A pesar de que los hombres, como hemos comentado anteriormente, suelen utilizar las redes para ver qué cuelgan sus contactos, cuando les preguntamos sobre cuáles eran los principales beneficios que para ellos ofrecian las herramientas, al igual que para las mujeres, respondieron que los dos beneficios principales eran estar en contacto con amigos y familiares y buscar información, seguido de conocer y manejar las nuevas tecnologías y ampliar sus conocimientos.
A pesar de que se va alcanzando la igualdad de género en cuanto al acceso, sí que es cierto que, aunque ambos géneros manifiestan no tener demasiado interés por las redes sociales, este es mucho menor en las mujeres que en los hombres

Igualmente, tanto hombres como mujeres coincidieron en que, entre sus menores beneficios de uso, está el hecho de conocer gente nueva.

No hemos encontrado diferencias entre los hombres y las mujeres mayores en relación con las herramientas que más utilizan habitualmente, siendo las más frecuentes, en ambos casos y en el mismo orden, Facebook, YouTube y Messenger. Sin embargo, en ambos géneros, son pocos los usuarios de herramientas como Twitter (red informacional en tiempo real que permite conectar con las últimas historias, noticias sobre lo que ocurre en el entorno o lo que están haciendo los sujetos), Windows Live, Wiki y Blog (donde los diferentes usuarios tienen la posibilidad de crear, añadir, modificar o borrar el contenido de dicha página web de forma colaborativa), Tuenti o Delicious. En definitiva, aún es elevado el número de herramientas que siguen siendo una asignatura pendiente en la formación del colectivo, independientemente del género. A este respecto, y como bien exponen Barroso y Aguilar (2015, pág. 19), «el reconocimiento de la necesidad de educación en las personas mayores se encuentra presente en el propio concepto de "educación permanente", donde se indica que la educación no debe ajustarse a un periodo concreto del devenir historiográfico, sino que todo ser humano debe continuamente ampliar sus conocimientos, habilidades y actitudes para no quedar obsoletos en una sociedad cambiante como en la que nos hallamos», por lo que es necesario ampliar las ofertas formativas al colectivo para poder solventar o aminorar el analfabetismo tecnológico existente en ellos. 
Por último, nos interesaba conocer igualmente si existía diferencia de género con respecto al dispositivo de conexión utilizado entre nuestras personas mayores. En este sentido, se observa una gran diferencia, ya que el dispositivo más utilizado por las mujeres es el portátil, mientras que en el caso de los hombres sigue siendo el ordenador fijo o de sobremesa. Aunque en los últimos años el número de usuarios que acceden a las redes sociales desde el teléfono móvil ha aumentado considerablemente en comparación con otros dispositivos de conexión, en el caso de las personas mayores sigue siendo muy reducido el número de sujetos que acceden a través de él, datos que coinciden con los resultados ofrecidos por Fundación Telefónica (2013), aunque, según nuestras conclusiones, a pesar de su escaso porcentaje de uso, este es menor en el caso de las mujeres que en el de los hombres. La PDA se convierte en el dispositivo de conexión prácticamente inutilizable por ambos géneros.

\section{BIBLIOGRAFÍA}

Abellán, A. y Pujol, R. [2015]: «Un perfil de las personas mayores en España, 2015. Indicadores estadísticos básicos», Informes Envejecimiento en Red, 10.

Abellán, A.; Vilches, J. y Pujol, R. [2014]: «Un perfil de las personas mayores en España, 2014. Indicadores estadísticos básicos», Informes Envejecimiento en Red, 6 .

Aguilar, S. y Benítez, R. [noviembre 2013]: «El adulto mayor ante las herramientas Web 2.0 o web social», XXVI Seminario Interuniversitario de Pedagogía Social, Oviedo.

Albert, M. ${ }^{a}$ J. [2006]: La investigación educativa: claves teóricas, Madrid: McGraw-Hill.

Aparici, R. y Osuna, S. [2013]: «La cultura de la participación», Revista Mediterránea de Comunicación, 4 (2), págs.137-148.

Barragán, R. y Ruiz, E. [2013]: «Brecha de género e inclusión digital. El potencial de las redes sociales en educación», Profesorado, 17 (1), págs. 309-323.

Barroso, J. y Aguilar, S. [2015]: «Las personas mayores y las redes sociales. Estudio de sus percepciones», Eticanet, 1 (15), págs. 16-36.

[2016]: «Las personas mayores y las redes sociales. Un análisis de la situación actual», Aula de Encuentro, 1 (18), págs. 228-250.

\section{Según los resultados de nuestro estudio, la finalidad con la que se conectan las personas mayores a las redes sociales es diferente según el género, siendo el principal motivo, para el hombre, el entretenimiento, centrado en conocer la información que cuelgan sus contactos, mientras que para la mujer esta sería su última finalidad}

Barroso, J. y Cabero, J. [2010]: La investigación educativa en TIC: visiones prácticas, Madrid: Síntesis.

Bell, C.; Fausset, C.; Farmer, S.; Nguyen, J.; Harley, L. y Bradley, W. [2013]: «Examining social media use among older adults», Proceedings of the 24th ACM Conference on Hypertext and Social Media (HT '13), ACM, New York (NY), USA, págs. 158-163. Disponible en: http://doi.acm.org/10.1145/2481492.2481509 [Consultado: 10 de septiembre de 2016].

Braun, M. T. [2013]: «Obstacles to social networking website use among older adults», Computers in Human Behavior, 29 (3), págs. 673-680.

Cabero, J. y Barroso, J. [2013]: «La utilización del juicio de experto para la evaluación de TIC: el coeficiente de competencia experta», Bordón, 65 (2), págs. 25-38.

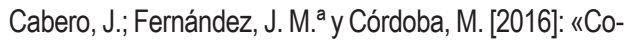
nocimiento de las TIC aplicadas a las personas con discapacidades. Construcción de un instrumento de diagnóstico», Magis. Revista Internacional de Investigación en Educación, 8 (17), págs.157-176.

Clercq, L. de [2009]: «¿Qué es la Web 2.0?», en M. Grané y C. Willem, C. (eds.), Web 2.0: nuevas formas de aprender y participar, Barcelona: Laertes, págs. 17-28. 
Colás, P.; González, T. y Pablos, J. de [2013]: «Juventud y redes sociales: Motivaciones y usos preferentes», Comunicar, XX (40), págs.15-23.

Corral, Y. [2009]: «Validez y confiabilidad de los instrumentos de investigación para la recolección de datos», Revista Ciencias de la Educación, 19 (33), págs. 229-247.

Dias, I. [2012]: «O uso das tecnologías digitais entre os seniores. Motivaçoes e intereses», Sociología, Problemas e Práticas, 68, págs. 51-77.

Edukanda [2016]: Sociedad del conocimiento y demografía. Importancia del perfil de usuario. Disponible en: http://www.edukanda.es/mediatecaweb/data/zip/1056/ curso3-Unidad1.pdf [Consultado: 10 de septiembre de 2016].

Fernández, M. ${ }^{a}$ y Fueyo, A. [2014]: «Redes sociales y mujeres mayores: estudio sobre la influencia de las redes sociales en la calidad de vida", Revista Mediterránea de Comunicación, 5 (1), págs.157-177.

Fundación Telefónica [2013]: La sociedad de la información en España 2012, Barcelona: Ariel.

Fundación Vodafone [2012]: TIC y mayores conectados al futuro. Disponible en: http://www.fundacionvodafone.es/publicacion/tic-y-mayores-conectados-al-futuro [Consultado: 10 de septiembre de 2016].

George, D. y Mallery, P. [2003]: Spss for Windows step by step: a simple guide and reference. 11.0 update, 4. ${ }^{\text {a }}$ ed., Boston: Allyn \& Bacon.

Giaroli, I. D.; González, S. y Martín, L. C. [2012]: «Ancianos: la audiencia de internet de la tercera edad en España», en J. P. Pérez (coord.), Estructura del mercado audiovisual: resultados. Disponible en: http:// www.eumed.net/librosgratis/2012b/1220/iancianos_ internet_tercera_edad_espana.html [Consultado: 10 de septiembre de 2016].

Gil, A.; Vitores, A.; Feliu, J. y Vall, M. [2011]: «Brecha digital de género: una revisión y una propuesta», TESI, 12 (2), págs. 25-53.

Hernández, R.; Fernández, C. y Baptista, P. [2006]: Meto-

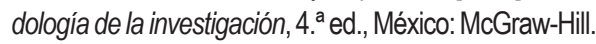

INE [2016a]: http://www.ine.es/ss/Satellite?L=es_ES\&c= INESeccion_C\&cid $=1259925530071 \& p=1254735110$ 672\&pagename=Productos YServicios\%2FPYSLayo ut\&param3=1259924822888 [Consultado: 10 de septiembre de 2016]. [2016b]: http://www.ine.es/ss/Satellite?L=es_ES\&c=IN ESeccion_C\&cid $=1259925528782 \& p=12547351106$ 72\&pagename=ProductosYServicios\%2FPYSLayout [Consultado: 10 de septiembre de 2016].

Instituto de la Mujer y para la Igualdad de las Oportunidades [2015]: Situación de la e-igualdad en España 2012 y 2013, Madrid: Instituto de la Mujer y para la Igualdad de Oportunidades.

Jackson, L. A.; Ervin, K. S.; Gardner, P. D. y Schmitt, N. [2001]: «Gender and the internet: women communicating and men searching», Sex Roles, 44 (5), pág. 363-379.

Jiménez, J. A. [2016]: Educación en nuevas tecnologías $y$ envejecimiento activo. Disponible en: http://www. educacionmediatica.es/comunicaciones/Eje\%203/ Juan\%20A.\%20Jim\%C3\%A9nez\%20L\%C3\%B3pez. pdf [Consultado: 8 de septiembre de 2016].

Martínez, R.; Delerue, A. y Silva, P. [2013]: «Mayores activos y su relación con internet: usos y motivaciones de uso en una muestra de mayores activos portugueses», Riadna, Cultura, Educación y Tecnología, 1 (1), págs.12-16.

Mateo, J. [2004]: «La investigación ex post-facto», en R. Bisquerra (coord.), Metodología de la investigación educativa, Madrid: La Muralla, págs.195-230.

O'Dwyer, L. y Bernauer, J. [2014]: Quantitative research for the qualitative researcher, Thousand Oaks (California): Sage.

OMS [2001]: «Salud y envejecimiento. Un documento para el debate», Boletín sobre el envejecimiento, perfiles y tendencias, 4 y 5, Imserso: Observatorio de personas Mayores.

Ortega, S. [2007]: «Evolución del perfil del usuario: usuarios 2.0.», No Solo Usabilidad: Revista sobre Personas, Diseño y Tecnología, 6. Disponible en: http://www.nosolousabilidad.com/articulos/usuario20. htm\#sthash.wQrkxGJH.dp uf [Consultado: 8 de septiembre de 2016].

Peral, B.; Arenas, J. y Ramón, M. Á. [2013]: «El papel de las variables sociodemográficas en el uso de las aplicaciones basadas en internet por los mayores», Innovar, 23 (48), págs. 55-65.

Requena, C.; Pastrana, I. M. y Salto, F. [2012]: «Multiplicadores de nuevas tecnologías», Cuadernos de la Cátedra Telefónica, (1), págs.15-26. 
Romero, R. [2011]: «Género en el uso del e-learning en las universidades andaluzas», Profesorado. Revista de Currículum y Formación de Profesorado, 15 (1), págs. 121-138.

Sabariego, M. [2004]: «El proceso de investigación», en R. Bisquerra (coord.), Metodología de la investigación educativa, Madrid: La Muralla, págs. 127-163.

Sebastián, A. y Martínez, G. [2013]: «La influencia de las nuevas tecnologías: videojuegos, redes sociales e internet, en los consumidores seniors en España», I Congreso Internacional de Comunicación y Sociedad, Logroño (La Rioja): Universidad Internacional de La Rioja.

Solano, I. M. y Bernal, R. M. [2011]: «Metodologías activas y redes sociales: configurando redes de colabo- ración en la educación superior», Congreso Internacional de Innovación Docente, Cartagena (Murcia): Universidad Politécnica de Cartagena.

Statista[2016]: https://es.statista.com/estadisticas/474930/ redes-sociales-numero-de-usuarios-espana/ [Consultado: 10 de septiembre de 2016].

Vilte, D.; Saldaño, V.; Martín, A. y Gaetán, G. [2013]: «Evaluación del uso de redes sociales en la tercera edad», Congreso Nacional de Ingeniería Informática/Sistemas de Información [en línea]. Disponible en: http://conaiisi.frc.utn.edu.ar/PDFsParaPublicar/1/schedConfs/7/142-446-1-DR.pdf [Consultado: 8 de septiembre de 2016].

Con este máster oficial, la UDIMA quiere formar profesores competentes que desarrollen una serie de cualidades, capacidades y competencias necesarias para desempeñar con eficacia su labor en entornos de docencia, gestión académica, diseño y edición de materiales y uso de las TIC, adaptando su actividad profesional a la demanda de los nuevos contextos de Enseñanza y Aprendizaje del Español como Lengua Extranjera (ELE). Este máster oficial [60 créditos ECTS] se inicia en octubre y febrero de cada año y su duración normal es de 12 meses.

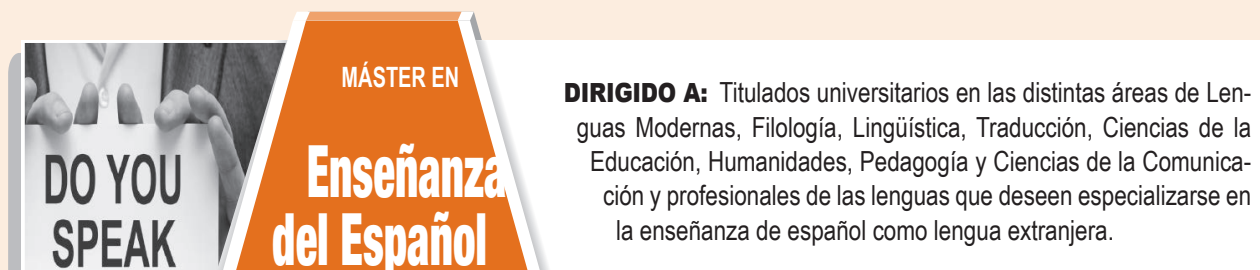

OBJETIVOS: Formar profesionales cualificados en el ámbito de la enseñanza del español como lengua extranjera, dotándoles de un perfil práctico y competitivo que les capacite para realizar adecuadamente y con garantía de éxito su labor profesional, facilitándoles su integración en un mercado laboral de enorme proyección internacional. 


\section{Magisterio de Educación Infantil}

Hoy en día los centros educativos tienen una imperiosa necesidad de disponer de personal especializado, capaz de hacer frente a las necesidades educativas de la etapa infantil, de acuerdo con los conocimientos ya logrados por las diversas ciencias que hoy se ocupan de los niños en los primeros años de su vida, así como de los logros relativos al desarrollo de la inteligencia, la emocionalidad y la formación de la personalidad temprana, resultantes de estudios recientes sobre el desenvolvimiento de la mente infantil.

PLAN DE ESTUDIOS (la obtención del grado conlleva la realización de 240 créditos)

\begin{tabular}{|c|c|c|c|}
\hline CURSO & ASIGNATURAS & TIPO & CRÉD. \\
\hline 1 & Didáctica e Innovación Curricular en Educación Infantil .............. & $\mathrm{T}$ & 6 \\
\hline 1 & Psicologia de la Educación & $\mathrm{T}$ & 6 \\
\hline 1 & Historia y Teoria de la Educación .................................................... & T & 6 \\
\hline 1 & Tecnologias de la Información y Gestión del Conocimiento ......... & $\mathrm{T}$ & 6 \\
\hline 1 & Psicologia del Desarrollo & $\mathrm{T}$ & 6 \\
\hline 1 & Desarrollo de Habilidades Lingüisticas y Lectoescritoras ............ & B & 6 \\
\hline 1 & 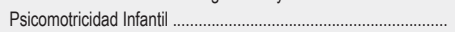 & B & 6 \\
\hline 1 & Sociologia de la Familia y de la Infancia ....................................... & $\mathrm{T}$ & 6 \\
\hline 1 & 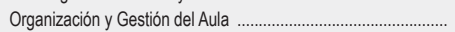 & $\mathrm{T}$ & 6 \\
\hline 1 & Métodos, Recursos y Nuevas Tecnologias para el Aprendizaje .. & $\mathrm{T}$ & 6 \\
\hline 2 & Conocimiento del Medio Social y Cultural y su Didáctica ............. & B & 6 \\
\hline 2 & Conocimiento del Medio Natural y su Didáctica ............................ & B & 6 \\
\hline 2 & 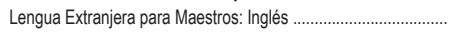 & B & 6 \\
\hline 2 & Psicologia del Aprendizaje & $\mathrm{T}$ & 6 \\
\hline 2 & 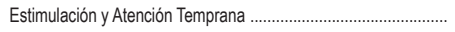 & $\mathrm{T}$ & 6 \\
\hline 2 & Sociología de la Educación & $\mathrm{T}$ & 6 \\
\hline 2 & Expresión Musical y su Didáctica ................................................. & B & 6 \\
\hline 2 & Desarrollo del Pensamiento Lógico Matemático y su Didáctica .. & B & 6 \\
\hline 2 & 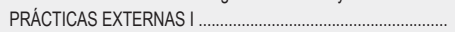 & B & 12 \\
\hline 3 & Lengua y Literatura y su Didáctica ............................................. & B & 6 \\
\hline 3 & 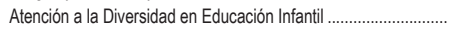 & $\mathrm{T}$ & 6 \\
\hline 3 & Didáctica de la Lengua Inglesa en Educación Infantil ................. & B & 6 \\
\hline 3 & 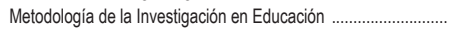 & $T$ & 6 \\
\hline 3 & 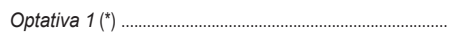 & 0 & 6 \\
\hline 3 & Organización y Liderazgo de Centros Escolares ............................... & $\mathrm{T}$ & 6 \\
\hline 3 & 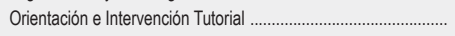 & $\mathrm{T}$ & 6 \\
\hline 3 & Optativa $2\left(^{*}\right)$ & 0 & 6 \\
\hline 3 & PRÁCTICAS EXTERNAS II & B & 12 \\
\hline 4 & 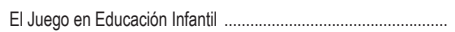 & $\mathrm{T}$ & 6 \\
\hline 4 & Expresión Plástica y Visual y su Didáctica ....................................... & B & 6 \\
\hline 4 & Optativa $3\left(^{*}\right)$ & 0 & 6 \\
\hline 4 & Optativa $4\left(^{*}\right)$ & 0 & 6 \\
\hline 4 & Optativa $5\left(^{*}\right)$ & 0 & 6 \\
\hline 4 & Salud, Infancia y Alimentación & $\mathrm{T}$ & 4 \\
\hline 4 & 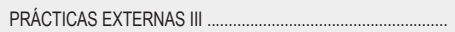 & B & 12 \\
\hline 4 & TRABAJO FIN DE GRADO & B & 14 \\
\hline
\end{tabular}

\section{Magisterio de Educación Primaria}

Son objetivos de la Educación Primaria, entre otros: conocer y apreciar los valores y las normas de convivencia, aprender a obrar de acuerdo con ellas, prepararse para el ejercicio activo de la ciudadanía y respetar los derechos humanos, así como el pluralismo propio de una sociedad democrática. También, desarrollar hábitos de trabajo individual y de equipo, de esfuerzo y responsabilidad en el estudio, así como actividades de confianza en uno mismo, sentido crítico, iniciativa personal, curiosidad, interés y creatividad en el aprendizaje.

PLAN DE ESTUDIOS (la obtención del grado conlleva la realización de 240 créditos)

\begin{tabular}{|c|c|c|c|}
\hline CURSO & ASIGNATURAS & TIPO & CRÉD. \\
\hline 1 & Didáctica e Innovación Curricular en Educación Primaria ............ & T & 6 \\
\hline 1 & 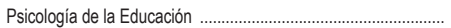 & T & 6 \\
\hline 1 & Historia y Teoria de la Educación & T & 6 \\
\hline 1 & Tecnologias de la Información y de Gestión del Conocimiento ... & B & 6 \\
\hline 1 & Psicologia del Desarrollo & $T$ & 6 \\
\hline 1 & 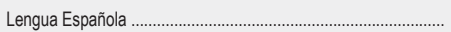 & $B$ & 6 \\
\hline 1 & 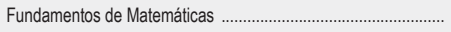 & B & 6 \\
\hline 1 & Conocimiento del Medio Natural .............................................. & B & 6 \\
\hline 1 & Sociologia de la Educación & $T$ & 6 \\
\hline 1 & Métodos, Recursos y Nuevas Tecnologias para el Aprendizaje .. & B & 6 \\
\hline 2 & 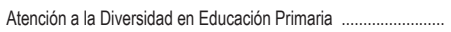 & $\mathrm{T}$ & 6 \\
\hline 2 & Educación Fisica y su Didáctica ..................................................... & B & 6 \\
\hline 2 & 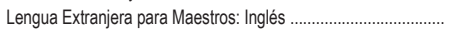 & B & 6 \\
\hline 2 & 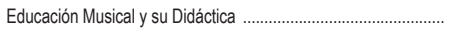 & B & 6 \\
\hline 2 & Metodologia de Investigación en Educación ………………........... & $\mathrm{T}$ & 6 \\
\hline 2 & Organización y Liderazgo de Centros Escolares ........................... & $\mathrm{T}$ & 6 \\
\hline 2 & 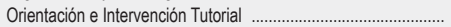 & $T$ & 6 \\
\hline 2 & Psicologia del Aprendizaje & $\mathrm{T}$ & 6 \\
\hline 2 & PRÁCTICAS EXTERNAS I & B & 12 \\
\hline 3 & Literatura Infantil y Juvenil & B & 6 \\
\hline 3 & 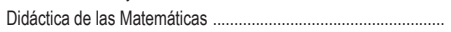 & B & 6 \\
\hline 3 & Didáctica de la Lengua Inglesa en Educación Primaria ................ & B & 6 \\
\hline 3 & 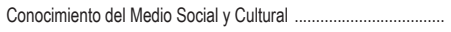 & B & 6 \\
\hline 3 & 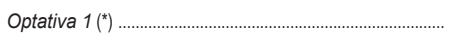 & 0 & 6 \\
\hline 3 & Didáctica de las Ciencias Sociales ............................................... & B & 6 \\
\hline 3 & Educación Plástica y Visual y su Didáctica ........................................ & B & 6 \\
\hline 3 & Optativa $2\left(^{*}\right)$ & 0 & 6 \\
\hline 3 & PRÁCTICAS EXTERNAS II & B & 12 \\
\hline 4 & 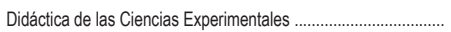 & B & 6 \\
\hline 4 & Didáctica de la Lengua y la Literatura ......................................... & $\mathrm{B}$ & 6 \\
\hline 4 & Optativa $3\left(^{*}\right)$ & 0 & 6 \\
\hline 4 & Optativa $4\left(^{*}\right)$ & 0 & 6 \\
\hline 4 & Optativa $5\left(^{*}\right)$ & 0 & 6 \\
\hline 4 & Educación en Valores & B & 4 \\
\hline 4 & 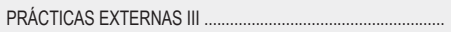 & B & 12 \\
\hline 4 & TRABAJO FIN DE GRADO & B & 14 \\
\hline
\end{tabular}

(*) La lista de asignaturas optativas se puede consultar en www.udima.es. $\mathrm{T}=$ Formación básica; $\mathrm{B}=$ Formación obligatoria; $\mathrm{O}=$ Asignatura optativa 


\section{Menciones en los grados de Magisterio}

\section{Menciones en los grados de Magisterio de Educación Infantil y Primaria}

Los grados en Magisterio de Educación Infantil y Primaria tienen cinco menciones. Cada una de ellas se compone de 30 créditos ECTS, pudiendo los estudiantes optar por una de las menciones para poder finalizar el grado o bien obtener el título sin mención cursando 30 créditos optativos a su libre elección de los ofertados. También podrían cursar más de una mención. Si el alumno deseara obtener varias menciones, deberá cursar los créditos asociados a cada una de ellas.

\begin{tabular}{|c|c|}
\hline \multicolumn{2}{|l|}{ Mención en Lengua inglesa } \\
\hline Asignatura & ETCS \\
\hline 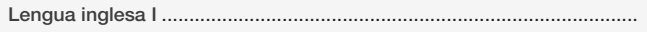 & 6 \\
\hline 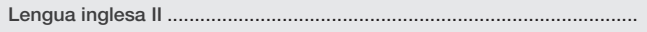 & 6 \\
\hline 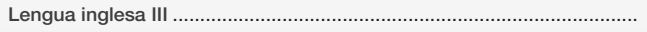 & 6 \\
\hline Educación para el bilingüismo: CLIL & 6 \\
\hline Didáctica avanzada de la lengua inglesa.. & 6 \\
\hline
\end{tabular}

\begin{tabular}{|c|c|}
\hline \multicolumn{2}{|l|}{ Mención en Pedagogía terapéutica } \\
\hline Asignatura & ETCS \\
\hline 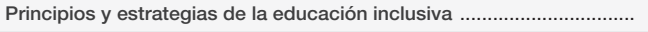 & 6 \\
\hline 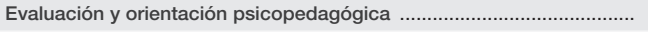 & 6 \\
\hline Necesidades específicas de apoyo educativo y orientación familiar & 6 \\
\hline Intervención psicopedagógica en dificultades de aprendizaje .............. & 6 \\
\hline $\begin{array}{l}\text { Programas de intervención para alumnos con necesidades específicas } \\
\text { de apoyo educativo }\end{array}$ & 6 \\
\hline
\end{tabular}

\begin{tabular}{|l|l|}
\hline \multicolumn{3}{|c|}{ Mención en Audición y lenguaje } \\
\hline Asignatura & ETCS \\
\hline
\end{tabular}

Principios y estrategias de la educación inclusiva................................... 6

Trastornos del habla y del lenguaje ..................................................... 6

Sistemas alternativos y aumentativos de comunicación ......................... 6

Evaluación e intervención en lenguaje oral.............................................. 6

Evaluación e intervención en lenguaje escrito ....................................... 6

\begin{tabular}{|c|c|}
\hline \multicolumn{2}{|l|}{ Mención en Tecnología educativa } \\
\hline Asignatura & ETCS \\
\hline 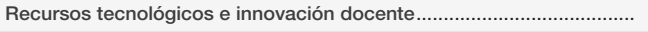 & 6 \\
\hline 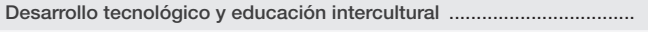 & 6 \\
\hline 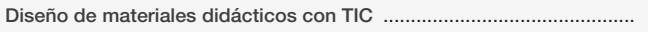 & 6 \\
\hline Integración de las TIC en la enseñanza de las artes y las humanidades & 6 \\
\hline Integración de las TIC en la enseñanza de las ciencias y las matemáticas & 6 \\
\hline
\end{tabular}

\begin{tabular}{|l|l|}
\hline \multicolumn{3}{|c|}{ Mención en Enseñanza de la religión católica* } \\
\hline Asignatura & ETCS \\
\hline
\end{tabular}

Historia de la Iglesia ...................................................................... 6

Religión, cultura y valores ............................................................... 6

El mensaje cristiano ...................................................................... 6

La Iglesia, los sacramentos y la moral ............................................. 6

Pedagogía y didáctica de la religión católica ......................................... 6

* Cubre los requisitos de formación universitaria para poder solicitar la DECA a la Conferencia Episcopal (ver plan de estudios).

\section{Curso de adaptación al grado}

Este curso de adaptación al grado ofrece a los maestros diplomados en la Especialidad de Educación Infantil o Primaria la posibilidad de obtener formación en campos determinados dentro del ejercicio profesional docente en estas etapas, a través de las menciones cualificadoras mencionadas anteriormente.

El objetivo principal del plan de estudios de este curso de adaptación al grado es contribuir a la actualización de la formación de los maestros diplomados. La aplicación de las TIC a la educación y de líneas pedagógicas innovadoras fruto de la investigación en educación hacen necesaria la actualización de los conocimientos didácticos de los diplomados y la formación de los maestros en investigación e innovación.

Al finalizar el curso de adaptación se obtiene el título de grado en Magisterio de Educación Infantil o de Educación Primaria.

La docencia en la etapa de educación infantil o primaria es una profesión regulada. Los graduados en Magisterio de Educación Infantil o Primaria tienen como principal salida profesional el trabajo como profesores en estas etapas, tanto en centros públicos como concertados y privados.

Si bien otras salidas profesionales para estos títulos pueden ser:

- Participación en proyectos educativos de organismos e instituciones (centros culturales, museos, asociaciones, ONG, etc.).

- Centros de educación para adultos.

- Centros de ocio y tiempo libre.

- Participación en programas de extensión educativa (actividades extraescolares, actividades de apoyo, etc.).

- Diseño y elaboración de materiales didácticos.

- Participación en proyectos de atención a la infancia y familiar. 


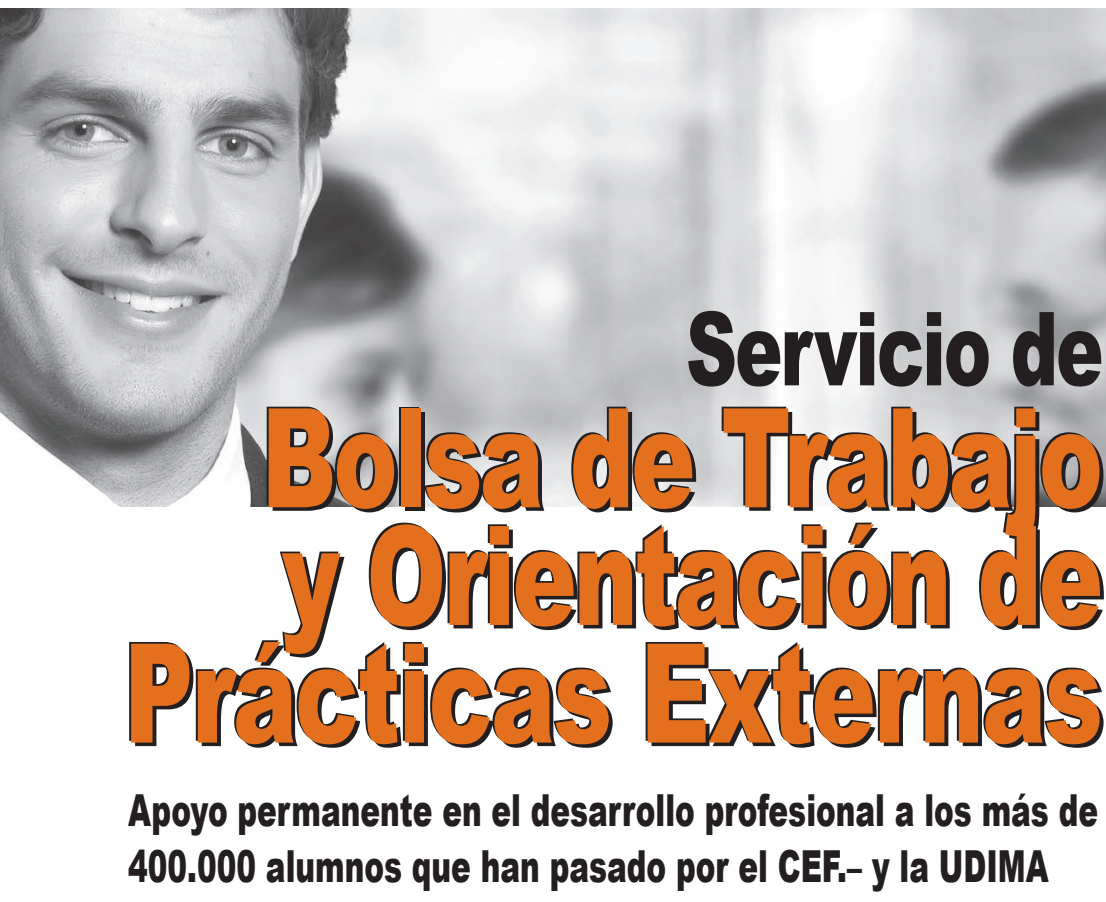

El EFF.- y la UDIMA ponen a disposición de los estudiantes diversos servicios de asesoramiento y formación para apoyar al alumno en su desarrollo académico y profesional. Es muy importante que el estudiante conozca la realidad laboral de su grado o máster, por lo que para finalizar sus estudios universitarios resulta imprescindible la realización de prácticas externas. Este servicio de Bolsa de Trabajo y Orientación de Prácticas Externas ofrece a las empresas y entidades educativas las herramientas necesarias para satisfacer sus necesidades en los procesos de selección de profesionales cualificados.

Desde nuestros orígenes hemos tenido como lema «formación para el empleo», por ello consideramos este servicio como un pilar fundamental para nuestras acciones formativas, pues todas van dirigidas a buscar empleo para nuestros alumnos, la mejora del que ya tienen 0 a conseguir su consolidación.
Desde el Servicio de Bolsa de Trabajo y Orientación de Prácticas Externas agradecemos tanto a las empresas y entidades educativas, como a los alumnos y antiguos alumnos que utilicen este servicio como fuente de reclutamiento para los procesos de selección en todas las áreas que se imparten en el EEF.- y en la UDIMA.

Este servicio es GRATUITO tanto para los estudiantes como para las entidades educativas.

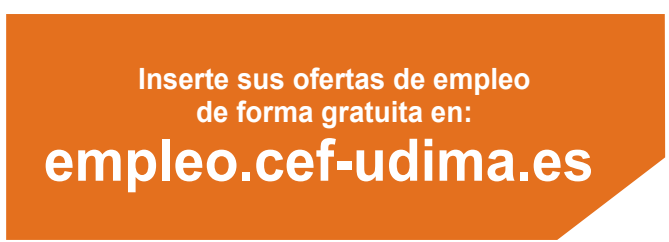

